\title{
The Ultraviolet Detection of Diffuse Gas in Galaxy Groups
}

\author{
John T. Stocke ${ }^{1}$, Brian A. Keeney ${ }^{1}$ (D), Charles W. Danforth ${ }^{1}\left(\mathbb{D}\right.$, Benjamin D. Oppenheimer $^{1}$ (1), Cameron T. Pratt ${ }^{1}$, \\ Andreas A. Berlind ${ }^{2}$ (D), Chris Impey ${ }^{3}$, and Buell Jannuzi ${ }^{3}$ \\ ${ }^{1}$ Center for Astrophysics and Space Astronomy, Department of Astrophysical and Planetary Sciences, University of Colorado, 389 UCB, Boulder, CO 80309, USA \\ ${ }^{2}$ Department of Physics and Astronomy, Vanderbilt University, PMB 401807, 2401 Vanderbilt Place, Nashville, TN 37240-1807, USA \\ ${ }^{3}$ Steward Observatory, University of Arizona, 933 N Cherry Avenue, Tucson, AZ 85721, USA \\ Received 2018 September 19; revised 2018 November 29; accepted 2018 December 3; published 2019 January 21
}

\begin{abstract}
A small survey of the UV-absorbing gas in 12 low-z galaxy groups has been conducted using the Cosmic Origins Spectrograph on board the Hubble Space Telescope. Targets were selected from a large, homogeneously selected sample of groups found in the Sloan Digital Sky Survey. A critical selection criterion excluded sight lines that pass close $(<1.5$ virial radii) to a group galaxy, to ensure absorber association with the group as a whole. Deeper galaxy redshift observations are used both to search for closer galaxies and also to characterize these $10^{13.5}-10^{14.5} M_{\odot}$ groups, the most massive of which are highly virialized with numerous early-type galaxies (ETGs). This sample also includes two spiral-rich groups, not yet fully virialized. At group-centric impact parameters of $0.3-2 \mathrm{Mpc}$, these signal-to-noise ratios $=15-30$ spectra detected $\mathrm{H} \mathrm{I}$ absorption in 7 of 12 groups; high (O VI) and low (Si III) ion metal lines are present in two-thirds of the absorption components. None of the three most highly virialized, ETG-dominated groups are detected in absorption. Covering fractions $\gtrsim 50 \%$ are seen at all impact parameters probed, but do not require large filling factors despite an enormous extent. Unlike halo clouds in individual galaxies, group absorbers have radial velocities that are too low to escape the group potential well without doubt. This suggests that these groups are "closed boxes" for galactic evolution in the current epoch. Evidence is presented that the cool and warm group absorbers are not a pervasive intra-group medium (IGrM), requiring a hotter $\left(T \sim 10^{6}-10^{7} \mathrm{~K}\right)$ IGrM to be present to close the baryon accounting.
\end{abstract}

Key words: galaxies: evolution - galaxies: groups: general - galaxies: halos - intergalactic medium - quasars: absorption lines

Supporting material: figure sets, machine-readable table

\section{Introduction}

In the past decade, the Hubble Space Telescope (HST) and its UV spectrographs have brought a tremendous increase in our knowledge of the relationship between galaxies and the diffuse circum- and intergalactic media (CGM and IGM) out of which galaxies are still forming. But while QSO absorptionline probes show that diffuse gas in the IGM accounts for the vast majority of baryons at all epochs (Rauch 1998; Shull et al. 2012), the "baryon census" is still not complete at low-z, either for individual spiral galaxies (Klypin et al. 2001; Stocke et al. 2013, although see Prochaska et al. 2017 for a different view) or on cosmological scales (Shull et al. 2012). With the IGM becoming more complex with time (e.g., Cen \& Ostriker 1999; Davé et al. 1999), the effects of galaxy feedback and nonequilibrium cooling complicate both an observational search and a theoretical prediction for diffuse gas associated with individual galaxies (Tumlinson et al. 2011; Oppenheimer et al. 2012; Stocke et al. 2013; Werk et al. 2013; Prochaska et al. 2017), galaxy groups and clusters (Yoon et al. 2012; Yoon \& Putman 2013, 2017), and large-scale galaxy structures (Wakker et al. 2015; Tejos et al. 2018).

Of particular interest in this regard are highly ionized metal (O VI and Ne VIII) lines and thermally broadened H I absorptions, which show that there is a sizable fraction of warm gas (following the naming convention in Savage et al. 2014, since sometimes gas in this temperature range is called the warm-hot IGM, or WHIM) locally at $T>10^{5} \mathrm{~K}$. The WHIM is suspected to harbor the bulk of the "missing baryons" at low- $z$, and may also be an important gas reservoir in the recycling processes of galactic winds and infall that continue to fuel ongoing star formation in spiral galaxies (e.g., Binney \& Tremaine 1987). Surveys for metal ion absorbers (e.g., Danforth \& Shull 2008; Steidel et al. 2010; Tumlinson et al. 2011; Burchett et al. 2016) show that gas enriched by star formation has been transported quite far from galaxies (Chen et al. 2001; Adelberger et al. 2005; Stocke et al. 2006, 2013; Prochaska et al. 2011; Johnson et al. 2015; Pratt et al. 2018), up to $\sim 600 \mathrm{kpc}$ or more in the current epoch, much larger than the inferred virial radius of even the most massive galaxies, but somewhat smaller than the size of most galaxy groups.

The science program of the Cosmic Origins Spectrograph (COS) Guaranteed Time Observers (GTOs; Stocke et al. 2013; Keeney et al. 2017) and the various HST guest observer projects, including COS-Halos, COS-Dwarfs and COS-GASS (Tumlinson et al. 2011; Borthakur et al. 2013; Werk et al. 2013; Bordoloi et al. 2014), and the Andromeda galaxy AMIGA project (Lehner et al. 2015), among others, have gone a long way toward defining the relationship between individual galaxies, their massive photoionized CGMs $\left(>10^{10} M_{\odot}\right.$ at $T \sim 10^{4} \mathrm{~K}$ for $L \geqslant L^{*}$, late-type galaxies), and the shocked interfaces with hotter gas, seen as O VI absorptions (Savage et al. 2010, 2012; Prochaska et al. 2011; Tumlinson et al. 2011, 2013; Thom et al. 2012; Borthakur et al. 2013; Stocke et al. 2013, 2014; Werk et al. 2013, 2014; Bordoloi et al. 2014; Lehner et al. 2014). However dramatic the CGM detections have been, these studies still leave $\lesssim 50 \%$ of spiral baryons unaccounted for in the current epoch (but see Prochaska et al. 2017 for an accounting in which the CGM baryon content is dominated by warm gas). It is possible that most, if not all, of the discrepancy in the baryon census found by these studies is due to 
differing assumptions involving the value for the low- $z$ extragalactic ionizing flux and the conversion between stellar mass and halo mass for massive spiral galaxies (Keeney et al. 2017). If there are as-yet undetected baryons in the CGM, this uncertainty leaves important questions unanswered, including those regarding the physical conditions of these baryons and if these baryons are still bound to the galaxies or blown out into the IGM.

Savage et al. (2014, Paper I hereafter) used HST/COS spectra with signal-to-noise ratio $(\mathrm{S} / \mathrm{N})=20-50$ to detect and analyze $54 \mathrm{O} \mathrm{VI}$ absorbers at low- $z$, including at least 14 systems that are demonstrably collisionally ionized gas at warm temperatures $\left(T>10^{5} \mathrm{~K}\right)$ too hot to be cool, photoionized gas. The remaining $\mathrm{O}$ VI systems are either cooler than this limit, suggesting photoionized gas, or have velocity misalignments that preclude conclusive temperature analysis (see Paper I for detailed methodology). The broad, shallow absorptions seen in many of these O VI plus Ly $\alpha$ systems are not obviously associated with individual galaxies, since many of these absorbers are found well outside the virial radius of the nearest galaxy (Keeney et al. 2017; Pratt et al. 2018).

In Stocke et al. (2014, Paper II hereafter), the galaxy environments of nearby $(z \leqslant 0.15)$ photoionized and collisionally ionized O VI absorbers from Paper I were investigated. Based on deep and wide galaxy surveys around these sight lines, groups of galaxies were found in all cases. Using a subset of these groups for which at least eight members were identified, weak correlations ( $\sim 95 \%$ confidence level) were found between the gas temperatures as inferred from the O VI and $\mathrm{HI} b$-values and the velocity dispersions and total luminosities of the groups. While an association between some of these absorbers and the nearest group galaxy to the sight line is plausible in several cases, Paper II puts forward the hypothesis that most of these warm absorbers are associated with an entire galaxy group.

If this is the case, a simple argument suggests that these warm absorbers are very large and massive enough to account for the remainder of the missing baryons in late-type galaxies; in other words, using the warm absorber line density of $d \mathcal{N} / d z \geqslant 4$ per unit redshift (Paper II) in conjunction with the local space density of galaxy groups (Pisani et al. 2003; Berlind et al. 2006) requires that these absorbers have radii $\geqslant 1 \mathrm{Mpc}$ at a high covering factor (larger still, if "patchy") and therefore are quite massive ( $\lesssim 10^{12} M_{\odot} ;$ Paper II). This is comparable to the mass of the X-ray emitting intra-cluster medium in elliptical-dominated groups and poor clusters, where it is the dominant baryon reservoir at halo masses of $\geqslant 10^{14.5} M_{\odot}$ (Mulchaey 2000).

However, little is known about the intra-group medium (IGrM) in the lower-mass halos $\left(10^{12}-10^{14} M_{\odot}\right)$ typical of small groups of galaxies, the most numerous ensembles of galaxies in the universe. If a massive IGrM is present at the expected temperatures $(\log [T / \mathrm{K}]=6.2-6.5$; Mulchaey et al. 1996), it would be difficult to detect-too cool to emit significantly at keV energies, and too hot to create narrow, high column density H I Ly $\alpha$ and metal absorption lines. But at the very least, a rudimentary (i.e., patchy and multi-temperature) IGrM is expected because disk galaxies merge to form the central elliptical in elliptical-dominated groups, which do exhibit massive, X-ray emitting IGrMs.

If an IGrM at warm temperatures or slightly higher is volume filling, its mass is comparable to the amount needed to bring groups up to the cosmic mean baryon-to-dark matter ratio (e.g., Klypin et al. 1999; McGaugh et al. 2000). This would make groups of galaxies, like the more massive clusters, "closed boxes" for galactic evolution. Recent observational work by Pratt et al. (2018) confirms earlier studies which showed that high metallicity gas $\left(Z \geqslant 0.1 Z_{\odot}\right.$ as traced by $\mathrm{O}$ VI absorption) does not extend farther than $\approx 600 \mathrm{kpc}$ from its source inside star-forming galaxies; this maximum distance that metal absorption (specifically O VI) extends from galaxies is insufficient for the gas to escape from the group. On the other hand, current numerical simulations differ by factors of three to five on the percentage of baryons retained by groups in this critical mass range $\left(\log \left[M / M_{\odot}\right]=13-14.5\right)$; for example, COSMO-Owls (Le Brun et al. 2014), EAGLE (Schaye et al. 2015), and ILLUSTRIS (Nelson et al. 2018) find divergent values in this halo mass range due to differing feedback prescriptions.

Reconciling the observational results with the numerical simulations is important. If all the baryons expelled from individual galaxies are retained inside galaxy groups, this would have important consequences for galactic evolution; for example, some of the observed width in the mass-metallicity relationship (Tremonti et al. 2004) could be due to differing chemical histories between groups of galaxies. Additionally, the continuing high star formation rate in spirals and the so-called G-dwarf problem (Binney \& Tremaine 1987; Pagel 2009) must be reconciled within individual galaxy groups. Given these important consequences, the hypotheses presented here, which are based on the HST/COS results of Papers I and II, need to be tested. This is one of the purposes of the current paper.

This paper is organized as follows. Section 2 describes the experimental design for this investigation and the selection of the sample of low- $z$ galaxy groups with QSO probes. Section 3 presents the HST/COS UV spectra and the basic analysis of the absorption systems detected at the redshifts of the foreground groups, including an analysis of the few possible broad Ly $\alpha$ absorbers (BLAs) in these groups. Section 4 presents the analysis of the galaxy redshift surveys conducted around each sight line based on the data found in Keeney et al. (2018), and describes the group-finding algorithm and derived group parameters (e.g., membership, sky position, redshift and velocity dispersion) for each group. Section 5 discusses the results of this survey of gas in galaxy groups, including whether the absorption is associated with an individual group galaxy or the entire group. Discussions of gas covering fractions, absorber kinematics, and absorber/group correlations are also included in this section. Section 6 summarizes the basic observational properties of this work and describes the important conclusions derived from this survey. Detailed descriptions of the $H S T / C O S$ spectroscopy are presented in Appendix A, including Table 10, which provides measurements for each absorption line detected that is associated with these groups. Appendix B presents detailed notes on each galaxy group. Throughout this paper, we assume a WMAP9 cosmology (i.e., $H_{0}=69.7 \mathrm{~km} \mathrm{~s}^{-1} \mathrm{Mpc}^{-1}, \Omega_{\Lambda}=0.718$, and $\Omega_{\mathrm{m}}=0.282$; Hinshaw et al. 2013).

\section{Experimental Design and Sample Selection}

This project investigates whether galaxy groups possess a cool and/or warm component to the IGrM by performing the reverse experiment to that of Papers I and II. In those papers, the $H S T / \mathrm{COS}$ spectra were used to find and identify WHIM absorbers; then the foreground galaxy distribution at the absorber distances were examined. Groups of galaxies were 
Table 1

HST/COS Observations

\begin{tabular}{|c|c|c|c|c|c|c|c|}
\hline $\begin{array}{l}\text { Group } \\
\text { ID } \\
(1)\end{array}$ & $\begin{array}{l}\text { AGN Target } \\
\text { Name } \\
(2)\end{array}$ & $\begin{array}{l}z_{\mathrm{AGN}} \\
(3)\end{array}$ & $\begin{array}{c}\text { Obs. Date } \\
\text { (4) }\end{array}$ & $\begin{array}{l}t_{\exp } \\
(\mathrm{ks}) \\
(5)\end{array}$ & $\begin{array}{c}F_{\mathrm{FUV}} \\
\left(10^{-15}\right) \\
(6)\end{array}$ & $\begin{array}{l}\mathrm{S} / \mathrm{N} \\
(7)\end{array}$ & $\begin{array}{l}\text { Notes } \\
\text { (8) }\end{array}$ \\
\hline 12833 & RBS 711 & 0.255 & $9 / 29 / 16$ & 5 & 12 & 29 & $14277-07$ \\
\hline 16803 & SDSS J1540-0205 & 0.321 & $7 / 3 / 16$ & 25.3 & $<1^{\mathrm{a}}$ & 11 & $14277-05,-06$ \\
\hline 19670 & SBS $0956+510$ & 0.143 & $6 / 23 / 16$ & 8.0 & 8 & 21 & $14277-09$ \\
\hline 25124 & B $1612+266$ & 0.395 & Jul/Aug $16^{\mathrm{b}}$ & 25.4 & 2 & 20 & $14277-03,-04$ \\
\hline 32123 & SDSS J1333+4518 & 0.320 & $4 / 26 / 17$ & 11 & 2 & 16 & $14277-16$ \\
\hline 36001 & SDSS J1028+2119 & 0.374 & $4 / 2 / 16$ & 15.9 & 4 & 26 & $14277-01,-02$ \\
\hline 44564 & CSO $1022^{c}$ & 0.285 & $6 / 30 / 14$ & 4.8 & 5 & 16 & $13444^{\mathrm{d}}$ (Wakker) \\
\hline 44565 & CSO $1022^{c}$ & 0.285 & $6 / 30 / 14$ & 4.8 & 5 & 16 & $13444^{\mathrm{d}}$ (Wakker) \\
\hline 44726 & CSO 1080 & 0.526 & $7 / 7 / 16$ & 10.1 & 5 & 19 & $14277-13,-15$ \\
\hline 44739 & FBQS J1519+2838 & 0.270 & $8 / 10 / 16$ & 15.8 & 3 & 22 & $14277-11,-12$ \\
\hline 44858 & FBQS J1519+2838 & 0.270 & $8 / 10 / 16$ & 15.8 & 3 & 22 & $14277-11,-12$ \\
\hline 50433 & FBQS J1030+3102 & 0.178 & $3 / 26 / 16$ & 4.9 & 12 & 23 & $14277-10$ \\
\hline
\end{tabular}

Notes.

a Partial LLS at source redshift mostly eliminates O VI sensitivity.

b Two coadded visits 2016 July 22 and August 6.

c Two groups are probed by the CSO 1022 sight line.

d Archival data set.

e Two groups are probed by the FBQS J1519+2838 sight line.

found to be present in virtually all cases. However, groups of galaxies are a very common phenomenon in the low- $z$ universe $\left(3 \times 10^{-3} \mathrm{Mpc}^{-3}\right.$ at $M_{\text {grp }} \geqslant 10^{13} M_{\odot} ;$ Berlind et al. 2006, Paper III hereafter). Moreover, these warm gas-selected groups in Paper II have rather heterogeneous properties, ranging from systems with total luminosities of $\sim 50 L^{*}$ and $\sigma_{\text {grp }} \approx 300-500$ $\mathrm{km} \mathrm{s}^{-1}$ to much smaller groups similar to the M51/M101 group. This diversity casts some doubt on the associations proposed in Paper II; in some cases, the absorption could be due to individual galaxy halos, not to gas associated with the entire group. Since galaxies and groups of galaxies are not independent phenomena, it is difficult to ascribe the association of gas to one and not the other using the method of Papers I and II.

Here we reverse this experiment. We start by pre-selecting a homogeneous set of low- $z$ galaxy groups defined by a uniform, objective selection method. By using a homogeneously selected sample of groups, we avoid the problem of assigning association to a very heterogeneous population for which a potential chance coincidence between absorbers and groups is possible. A large catalog of galaxy groups $(>7500)$ chosen systematically from the Sloan Digital Sky Survey (SDSS) using the algorithm of Paper III was used to identify appropriate groups for study.

Restricting the groups searched to redshifts of $z=0.1-0.2$ (1) allows good SDSS galaxy group membership selection and characterization (e.g., estimated total luminosities, sizes and velocity dispersions); (2) facilitates excellent follow-up observations using multi-object galaxy spectroscopy (MOS already in-hand; Keeney et al. 2018); and (3) allows both the O VI doublet and $\operatorname{Ly} \alpha$ (as well as $\operatorname{Ly} \beta$, Si III, and other lines of potential interest) to be observed with $\operatorname{COS}$ in the highest throughput G130M mode in a minimum number of $H S T$ orbits. Much of this wavelength range is blueward of the Ly $\alpha$ rest wavelength, making an O VI identification much easier and more secure, even if only one line of the doublet is detected (Stocke et al. 2017).
Next, we cross-correlated the redshift-restricted group catalog with a list of bright $(V \lesssim 17.5)$ background QSO targets, which allows high-S/N spectra to be obtained in just a few orbits. We required that the AGN sight lines intersect these groups at a range of impact parameters $(\rho): 0.25 \leqslant \rho \leqslant 1.5$ group virial radii (symbolized $R_{\text {grp }}$ herein to distinguish this scaling parameter from an individual galaxy's virial radius, $\left.R_{\mathrm{vir}}\right)$. Some of these impact parameters are modified by our more detailed examination of group membership and properties described in Section 4. While sight lines at larger impact parameters were available, association between absorbers and individual groups become less secure at larger radii. Importantly, we rejected sight lines that passed within $1.5 R_{\text {vir }}$ of a group galaxy to make sure we were observing group gas, not gas associated with individual galaxies (see, e.g., Prochaska et al. 2011; Stocke et al. 2013; Keeney et al. 2017). An initial evaluation of sight line "isolation" using the SDSS spectroscopic survey finds no proximate galaxies at $L \geqslant 0.9-2.5 L^{*}$ (limit depends on the specific group redshift). Sight line isolation is reevaluated in Section 4 using a considerably deeper galaxy redshift survey conducted after the HST/COS observations were secured. This reevaluation finds two groups (25124 and 32123) with galaxies close to the sight line, which removes one or more of the detected absorption systems from consideration as group gas.

The resulting QSO target list, along with basic observational details, is shown in Table 1. Despite having thousands of groups in the catalog from which to choose, the restrictions mentioned here leave only about two dozen, which have bright QSOs projected nearby. Only six of these targets had been previously observed by COS and all but one of these six have $\mathrm{S} / \mathrm{N}$ too low for detecting broad, shallow WHIM absorbers.

However, FBQS $1010+3003$ has medium-quality archival COS data $(\mathrm{S} / \mathrm{N}=15$ (20) at O VI (Ly $\alpha)$; Stocke et al. 2017) with a broad $\left(b \approx 100 \mathrm{~km} \mathrm{~s}^{-1}\right)$, shallow, $4 \sigma$ detection of $\mathrm{O}$ VI $1032 \AA$ at approximately the redshift of a group in our catalog. While Ly $\alpha$ shows several narrower, photoionized CGM components most likely associated with individual group 
Table 2

Group Properties from SDSS Analysis

\begin{tabular}{|c|c|c|c|c|c|c|c|c|}
\hline $\begin{array}{l}\text { Group } \\
\text { (1) }\end{array}$ & $\begin{array}{l}N_{0} \\
(2)\end{array}$ & $\begin{array}{l}\text { R.A.0 } \\
\text { (3) }\end{array}$ & $\begin{array}{l}\text { Decl. }_{0} \\
\text { (4) }\end{array}$ & $\begin{array}{c}z_{0} \\
(5)\end{array}$ & $\begin{array}{c}\log M_{0} \\
\text { (6) }\end{array}$ & $\begin{array}{l}\sigma_{0} \\
(7)\end{array}$ & $\underset{(8)}{\text { R.A.* }}$ & $\underset{(9)}{\text { Decl. }^{*}}$ \\
\hline 12833 & 8 & $129.423 \pm 0.040$ & $44.295 \pm 0.029$ & $0.14693 \pm 0.00077$ & $14.42 \pm 0.26$ & 534 & 129.245433 & 44.433969 \\
\hline 16803 & 10 & $234.981 \pm 0.015$ & $-2.040 \pm 0.040$ & $0.14839 \pm 0.00046$ & $14.54 \pm 0.25$ & 586 & 235.081530 & -2.084831 \\
\hline 19670 & 8 & $150.196 \pm 0.044$ & $50.794 \pm 0.028$ & $0.13443 \pm 0.00052$ & $14.38 \pm 0.26$ & 518 & 149.881960 & 50.746969 \\
\hline 25124 & 7 & $243.541 \pm 0.018$ & $26.625 \pm 0.036$ & $0.18613 \pm 0.00038$ & $14.64 \pm 0.24$ & 632 & 243.544250 & 26.547278 \\
\hline 32123 & 6 & $203.074 \pm 0.066$ & $45.413 \pm 0.024$ & $0.15971 \pm 0.00044$ & $14.15 \pm 0.29$ & 434 & 203.253458 & 45.302500 \\
\hline 36001 & 3 & $157.110 \pm 0.045$ & $21.338 \pm 0.028$ & $0.18788 \pm 0.00023$ & $14.17 \pm 0.28$ & 441 & 157.060646 & 21.331967 \\
\hline 44564 & 4 & $208.120 \pm 0.040$ & $36.261 \pm 0.022$ & $0.14506 \pm 0.00050$ & $14.04 \pm 0.30$ & 399 & 208.358845 & 36.347070 \\
\hline 44565 & 5 & $208.151 \pm 0.016$ & $36.280 \pm 0.013$ & $0.14925 \pm 0.00054$ & $14.21 \pm 0.28$ & 455 & 208.358845 & 36.347070 \\
\hline 44726 & 4 & $226.175 \pm 0.034$ & $29.791 \pm 0.035$ & $0.15208 \pm 0.00092$ & $13.76 \pm 0.35$ & 322 & 226.365002 & 29.788439 \\
\hline 44739 & 7 & $229.811 \pm 0.033$ & $28.413 \pm 0.051$ & $0.11839 \pm 0.00023$ & $14.22 \pm 0.28$ & 458 & 229.900610 & 28.641011 \\
\hline 44858 & 9 & $230.091 \pm 0.027$ & $28.850 \pm 0.013$ & $0.12740 \pm 0.00055$ & $14.44 \pm 0.26$ & 542 & 229.900610 & 28.641011 \\
\hline 50433 & 4 & $157.742 \pm 0.026$ & $30.864 \pm 0.009$ & $0.13599 \pm 0.00033$ & $14.12 \pm 0.29$ & 424 & 157.746220 & 31.048811 \\
\hline
\end{tabular}

Note. All coordinates are $\mathrm{J} 2000, M_{0}$ has units of $M_{\odot}$, and $\sigma_{0}$ has units of $\mathrm{km} \mathrm{s}^{-1}$. R.A.` and decl.» are the coordinates of the COS sight line.

galaxies, there is no Ly $\alpha$ absorber that can be associated with the O VI. This requires $T>10^{6} \mathrm{~K}$, as in Savage et al. (2010). There is no other plausible identification for the O VI line, since it is found blueward of the Ly $\alpha$ rest wavelength.

Based on an analysis of 5 SDSS galaxies and 15 from WIYN/HYDRA (Keeney et al. 2018) located at $\rho<2 \mathrm{Mpc}$ and $\left|\Delta v_{\text {abs }}\right|<2000 \mathrm{~km} \mathrm{~s}^{-1}$ from the O VI absorber, this group has $\sigma_{\text {grp }} \approx 200 \mathrm{~km} \mathrm{~s}^{-1}$ (Stocke et al. 2017), similar to what was found by Paper II for some absorber-selected galaxy groups. In this case, the group centroid on the sky and its mean recession velocity made a group association with the OVI absorber unlikely. Instead, Stocke et al. (2017) concluded that the warm gas detection most likely was associated with the single, nearest galaxy to the absorber. This test case is a cautionary tale for this project, highlighting both the importance of choosing targets well away from bright group galaxies, and also the difficulty in both characterizing groups of galaxies and determining if the observed UV absorption system is related primarily to an individual galaxy or to the entire group.

With the absence of sufficient high-S/N UV spectra in the $H S T$ archive to conduct this experiment, we used the SDSS group catalog to identify $10 \mathrm{new}$, bright targets that span a range of impact parameters through low $z$ galaxy groups. Of the 10 targets selected, one (CSO 1022) was observed by Program 13444 (PI: Wakker) during the previous cycle. These 10 QSO sight lines plus the FBQS $1010+3003$ sight line probe a well-defined sample of 13 pre-selected galaxy groups at a variety of impact parameters (two sight lines probe two distinct groups at two different redshifts; see Table 1). In conjunction with the "serendipitous" sample of group WHIM detections from Papers I and II, these detections either will affirm or deny the proposed model for these warm absorbers as an IGrM.

Table 2 lists the properties of the groups defined by the SDSS analysis of Paper III. The group identifier is listed in column 1, followed by the number of group members in column 2. The group centroid on the sky is listed in columns 3 and 4 , its redshift in column 5 , and its estimated mass $\left(M_{0}\right)$ and velocity dispersion $\left(\sigma_{0}\right)$ in columns 6 and 7. Finally, the coordinates of the QSO sight line that probes the group (Table 1) are listed in columns 8 and 9 . Note that despite the modest numbers of group members $\left(N_{0}=3-10\right)$, these groups have $M_{0}>10^{14} M_{\odot}$ in most cases.

\section{HST/COS Spectroscopy}

This section details the UV absorption systems associated with the 13 SDSS galaxy groups. Systems of absorption are considered within $\pm 2.5 \sigma_{0}$ of the mean redshift of the group (see Table 2). This broad window is necessary both because individual components of the system may be related either to an individual galaxy or the entire group and also because the original group mean position and recession velocity were based on just the few brightest SDSS group galaxies. We revise the original estimate of group parameters using SDSS combined with the MOS presented in Section 4, and will revisit the velocity, velocity dispersion, and sky offsets of each group as it pertains to the detected absorbers.

For each of the nine sight lines in our HST observing program (\#14277: Stocke, PI), a single COS G130M spectrum was obtained over several orbits in HST Cycle 23 (see Table 1 for details). The G130M spectrum of CSO 1022 was taken by program \#13444 in the previous HST cycle and obtained $\mathrm{S} / \mathrm{N} \approx 16$ at $1200 \AA$. The G130M spectrum of FBQS 1010 +3003 exceeds this criteria at $\mathrm{Ly} \alpha$ and is close to this $\mathrm{S} / \mathrm{N}$ ratio at $\mathrm{O}$ VI (as discussed previously).

All other sight lines have a $\mathrm{S} / \mathrm{N}$ ratio at this level or greater, allowing the $4 \sigma$ detection of an unresolved O VI $1032 \AA$ line with an equivalent width $W_{\lambda} \gtrsim 40 \mathrm{~m}$, corresponding to an $\mathrm{O} \mathrm{VI}$ column density of $N_{\mathrm{O} \text { VI }} \gtrsim 10^{13.5} \mathrm{~cm}^{-2}$ in the coadded spectra. However, one spectrum obtained by this program, SDSS J1540 -0205, has a higher redshift Lyman limit system (LLS) that obscures the wavelength where the OVI absorption would appear in group $16803\left(z_{0}=0.14839\right)$. Because Si III absorption is not detected in a high-S/N portion of this same spectrum at the group redshift, we conservatively identify this sight line as an HI-only detection, even though the potential O VI wavelength does not meet the provided criteria.

Spectra were processed using the techniques employed in Danforth et al. (2016), including automated line-identification procedures based on the Faint Object Spectrograph procedure developed by Schneider et al. (1993), with statistical significance for each line determined by the method developed specifically for the COS data by Keeney et al. (2012). In our first analysis of each spectrum, we searched for systems of absorbers (see detailed discussion of systems versus components in Danforth et al. 2016) and then refit each spectrum to 
Table 3

Detections/Non-detections in Group Sight Lines

\begin{tabular}{|c|c|c|c|}
\hline $\begin{array}{l}\text { Group } \\
\text { (1) }\end{array}$ & $\begin{array}{l}\rho / R_{0} \\
\quad(2)\end{array}$ & $\begin{array}{l}\text { AGN } \\
(3)\end{array}$ & $\begin{array}{l}\text { Absorption Component Notes } \\
\text { (4) }\end{array}$ \\
\hline 12833 & 0.9 & RBS711 & Strong H I; no O VI \\
\hline 16803 & 0.5 & SDSS15403 & $\begin{array}{l}\text { Weak H I at }-1036 \mathrm{~km} \mathrm{~s}^{-1} \text {; partial LLS } \\
\text { blocks O VI }\end{array}$ \\
\hline 19670 & 0.9 & SBS0956 & Strong $\mathrm{H} \mathrm{I}$; no O VI \\
\hline 25124 & 0.4 & B1612 & $\begin{array}{l}2 \text { strong H I, } 1 \text { possible BLA; O VI aligned } \\
\text { with BLA }\end{array}$ \\
\hline 32123 & 1.1 & SDSS1333 & $\begin{array}{l}3 \text { strong H I, } 1 \text { weak BLA; } 1 \text { weak O VI; } 1 \\
\text { Si III }\end{array}$ \\
\hline 36001 & 0.3 & SDSS 1028 & $\begin{array}{l}3 \text { strong H I; } 1 \text { weak O VI; } 1 \text { Si III; probably } \\
\text { aligned }\end{array}$ \\
\hline 44564 & 1.3 & CSO1022 & Nothing \\
\hline 44565 & 1.0 & CSO1022 & Nothing \\
\hline 44726 & 1.4 & CSO1080 & Strong $\mathrm{H} \mathrm{I}$ at $+1200 \mathrm{~km} \mathrm{~s}^{-1}$; no O VI \\
\hline 44739 & 1.1 & FBQS1519 & $\begin{array}{l}4 \text { strong } \mathrm{H} \mathrm{I} ; 2 \text { weak O VI, } 1 \text { with } \mathrm{Si} \text { III; } \\
\text { probably aligned }\end{array}$ \\
\hline 44858 & 1.1 & FBQS1519 & $\begin{array}{l}\text { Strong H I; moderate O VI; slight } \\
\text { misalignment }\end{array}$ \\
\hline 50433 & 1.1 & FBQS1030 & Nothing \\
\hline
\end{tabular}

Note. Column 2 lists the impact parameter between the SDSS group barycenter and the QSO sight line, normalized by the group virial radius. More details on group properties from the SDSS analysis can be found in Table 2.

determine the component(s) associated with the O VI absorption, if present.

We present a top-level summary of the absorption-line findings in Table 3. Four sight lines (five groups) show strong $\mathrm{HI}$ absorption and significant (though not necessarily strong) $\mathrm{O}$ VI absorption in at least one component. Four sight lines show HI absorption, but no O VI; this includes the SDSSJ 15403-0205 sight line, where a weak Ly $\alpha$ feature is seen $\sim 900 \mathrm{~km} \mathrm{~s}^{-1}$ from the SDSS center of group 16803, but the corresponding $\mathrm{O}$ VI is obscured by an intervening LLS. Finally, two sight lines (three groups) show no significant absorption of any kind within the established velocity range of the group (see Section 5.1).

Figure 1 shows all absorption within $\pm 2.5 \sigma_{0}$ of the SDSS group redshifts in Table 2, indicated by the green circles, whose error bars indicate the SDSS redshift uncertainty. The blue diamonds with error bars show the revised group redshifts and uncertainties from Table 6 (see Section 4.3 for details). Red dashed lines and labels indicate absorption near the group redshift, while gray labels indicate absorption at other redshifts, including Galactic lines. The galaxy symbol indicates the velocity of the nearest galaxies within $1.5 R_{\mathrm{vir}}$ of the sight line (3 cases only).

Discussions of the absorption toward each sight line are presented in Appendix A, including Table 10, which details line measurements of all $\mathrm{HI}$ and $\mathrm{O}$ VI lines detected within $\pm 2.5 \sigma_{0}$ of the SDSS group centers. In many cases, particularly for absorbers that fall at large velocity offsets from the group, these systems may be coincidental and not associated with the galaxy groups. We list these line parameters for completeness. The limiting factor on the velocity accuracy for individual absorbers, especially Ly $\alpha$ absorbers, is often set by the ability to de-convolve complex line profiles into individual components. A complete discussion of the line-identification and measurement techniques is found in Danforth et al. (2016).

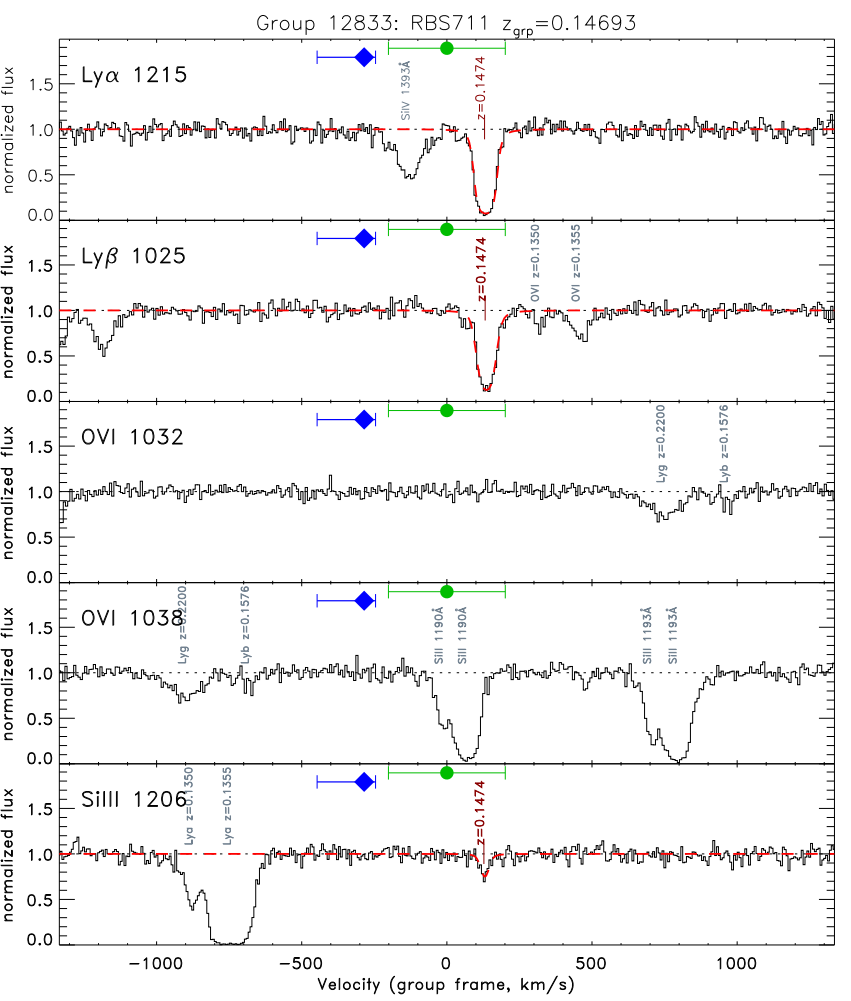

Figure 1. H I, O VI, and Si III absorption within $\pm 2.5 \sigma_{0}$ of group 12833. The panels are centered on the SDSS group redshifts (green circles) and use the SDSS velocity dispersion, $\sigma_{0}$, from Table 2 . Blue diamonds show the group redshift and its uncertainty derived from our updated Monte Carlo analysis (Table 6). Strong, narrow $\mathrm{H} \mathrm{I}$ is seen at $\Delta v_{\mathrm{abs}}=+129 \mathrm{~km} \mathrm{~s}^{-1}$ with no corresponding O VI. A weak Si III feature aligned with the $\mathrm{H}$ I absorption shows the existence of enriched, photoionized gas. Absorption features unrelated to the galaxy group are identified in gray with either wavelengths (for Galactic features) or redshifts (for unrelated IGM lines).

(The complete figure sets (11 images) is available.)

\subsection{Metal-line Absorbers}

As listed in Table 10 (see Appendix A) and shown in Figure 1, metal lines are detected in two-thirds (12 out of 18) of the absorption components. O VI is detected in seven of the metal-bearing systems as expected (Pratt et al. 2018), given the large impact parameters from individual galaxies designed into this study. The remaining absorbers contain significant low-ion absorption, usually Si III $1206 \AA$, similar to the photoionized absorbers in the CGM of individual galaxies. In the following discussion, the individual absorption components will be labeled by: [group/Ly $\alpha$ redshift].

Because this study was able to observe these targets only with COS/G130M, the higher ionization transitions normally seen in photoionized gas ( $\mathrm{Si}$ IV and C IV) are not available, making a robust ionization model impossible for these absorbers. In lieu of that preferred solution, we assume that the five absorbers with only low ions (usually Si III) are close to photoionization equilibrium (PIE), with a characteristic gas temperature of $\sim 20,000 \mathrm{~K}$ as a very rough estimate. These absorbers are 12833/0.14743, 25124/0.18528, 32123/ $0.15874,36001 / 0.18585$ (C III is the only metal line detected), and 44739/0.11706. Three absorbers are detected in H I and $\mathrm{O}$ VI only, and have O VI and H I line widths consistent with warm gas; their characteristic temperatures are derived in Section 3.2. 
This leaves four absorbers with both Si III and O VI detected. For three (32123/0.16081, 36001/0.18527, and 44739/0.11537) of these four composite systems, both the O VI and Si III are too weak to provide any definitive information, so the temperatures of these absorbers are poorly estimated. By our automated measurement, the first two of these three absorbers have $b_{\mathrm{O} \text { VI }}>b_{\mathrm{HI}}$, an unphysical situation if the $\mathrm{HI}$ and $\mathrm{O}$ VI reside in the same gas. Although the error bars on these measurements do overlap, the more straightforward solution is that the narrow $\mathrm{HI}\left(b \approx 25 \mathrm{~km} \mathrm{~s}^{-1}\right)$ is not co-located with the broader $\mathrm{O} \mathrm{VI}$ $\left(b \approx 30 \mathrm{~km} \mathrm{~s}^{-1}\right)$, but is associated with the Si III absorption.

We suspect that a BLA is present in the saturated Ly $\alpha$ profile that matches the OVI line widths in these two cases but the $\mathrm{O}$ VI profiles are too weak to provide tight enough constraints to specify the BLA parameters. Instead, we can set only very loose upper limits on the temperature of these two absorbers by assuming that the $\mathrm{OVI}$ line widths are entirely thermal: $T<8 \times 10^{5} \mathrm{~K}$ and $T<1 \times 10^{6} \mathrm{~K}$, respectively. This assumption leaves the observed Si III lines and the narrow $\mathrm{H}$ I lines as low temperature, photoionized absorbers.

For the third system in this list $(44739 / 0.11537)$, $b_{\mathrm{HI}}>b_{\mathrm{OVI}}$ and $\mathrm{Si}$ III is much stronger than the O VI. In this case, a photoionization model seems plausible and no BLA is required. In the fourth absorber (44739/0.11766), the O VI is very broad and symmetrical, suggesting that a BLA is present and the absorber temperature is $>10^{5} \mathrm{~K}$ (see Section 3.2).

For the metal-free absorbers, no definitive ionization-state or temperature determination is possible, except for absorber $32123 / 0.16142$, which has a very broad and symmetrical Ly $\alpha$ profile with a line width suggesting a temperature in CIE just barely exceeding $10^{5} \mathrm{~K}$. As discussed previously (Stocke et al. 2013; Werk et al. 2014; Keeney et al. 2017), absorbers with no metal lines detectable in these spectra means only that the metallicities are low, typically $\lesssim 10 \%$ Solar metallicity, so that some metals may well be present in these six "metal-free" absorbers as well.

\subsection{Presence of BLAs}

The spectra shown in Figure 1 and absorptions described in Table 10 use the automated line-finding and measurement program described in Danforth et al. (2016). However, that routine is not well-suited to the detection of broad, shallow components in complex Ly $\alpha$ profiles, which can indicate the presence of warm-hot gas in these groups. Since Paper II found evidence linking systems containing broad BLAs as well as broad, shallow O VI absorption with galaxy groups, it is important to establish how many absorbers found here are consistent with possessing BLAs and thus with warm-hot gas in these groups.

To investigate the possibility that BLAs are present in these spectra, the three Ly $\alpha$ lines were refit for cases in which the $\mathrm{O}$ VI absorption suggests the presence of warm gas (see the procedure developed in Papers I and II using the H I and O VI absorptions only). Briefly, the velocity and $b$-value of the $\mathrm{O}$ VI lines are used to constrain the wavelength and width of a potential BLA. The high-S/N ratio of these spectra is a necessity for this procedure.

Group 25124. The presence of a BLA is suggested in this case by an offset in the velocity centroid between the strong, broad, symmetrical O VI 1038 line compared to the two H I components and low-ionization metal ions. The stronger O VI line is blended with a Ly10 absorption at $z=0.330$ (see
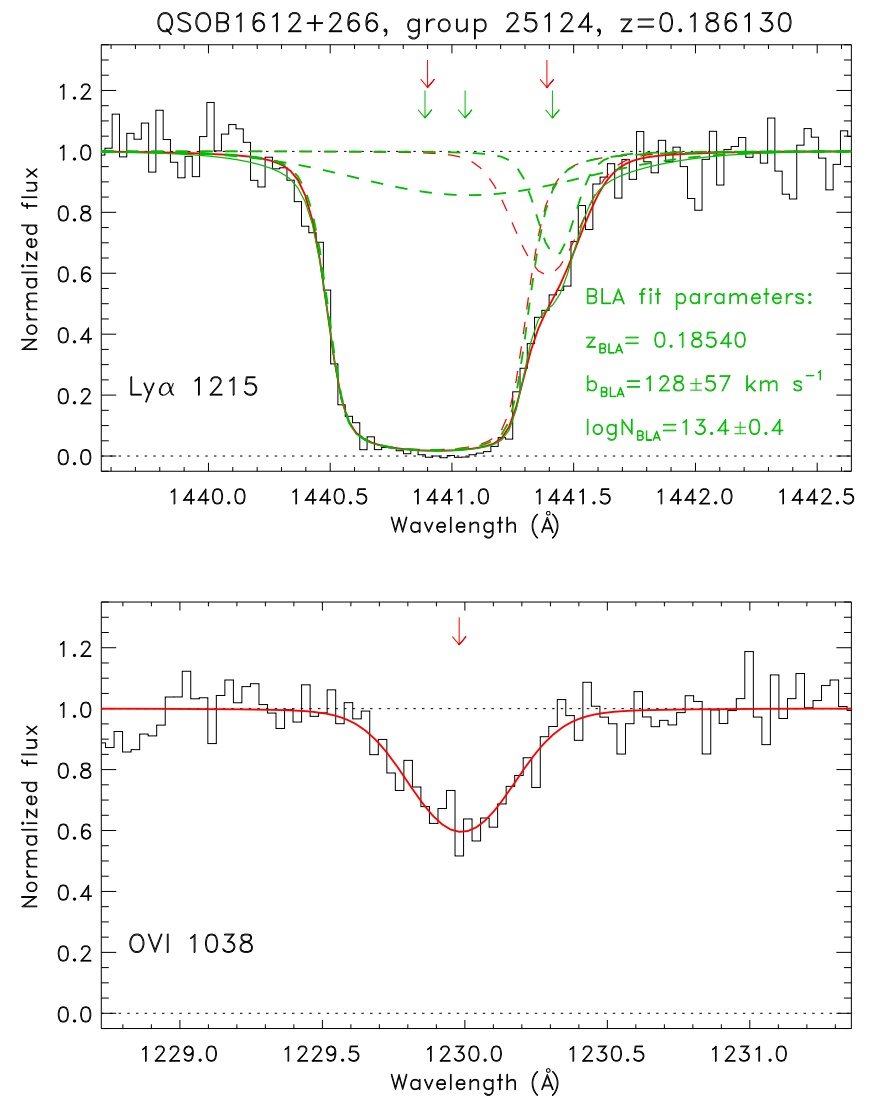

Figure 2. Probable BLA in the absorption associated with group 25124. Nominal fits are shown in solid red lines for Ly $\alpha$ and O VI $1038 \AA$ (the O VI $1032 \AA$ line is blended with Ly10 at a different redshift as discussed in the text) with red arrows marking the velocities. Individual components are shown as dashed lines. The green line shows the Ly $\alpha$ profile refitted with a third component centered at the same velocity as the broad, symmetrical O VI absorber; individual velocity components for this second fit are shown as dashed lines. The green arrows indicate the positions of the new Ly $\alpha$ components. The BLA has best-fit parameters shown in the plot, with a large $b$-value implying warm gas temperatures $\left(T \approx 8 \times 10^{5} \mathrm{~K}\right.$, but with considerable uncertainty).

Table 4

Probable BLA in Group 25124

\begin{tabular}{cccccc}
\hline \hline & $\begin{array}{c}\lambda \\
(\AA)\end{array}$ & $z$ & $\begin{array}{c}b \\
\left(\mathrm{~km} \mathrm{~s}^{-1}\right)\end{array}$ & $\begin{array}{c}\mathrm{EW} \\
(\mathrm{m \AA})\end{array}$ & $\begin{array}{c}\log N \\
(N \text { in cm }\end{array}$ \\
\hline$\# 1$ & 1440.89 & 0.18526 & $37 \pm 4$ & 708 & $15.89 \pm 0.41$ \\
$\# 2$ & 1441.05 & 0.18540 & $128 \pm 57$ & 140 & $13.43 \pm 0.38$ \\
$\# 3$ & 1441.41 & 0.18569 & $18 \pm 7$ & 63 & $13.15 \pm 0.19$ \\
\hline
\end{tabular}

Figure 1, online panel 4). Using the location and width of the O VI $1038 \AA$ line, the Ly $\alpha$ profile was refit, and a BLA matching the O VI can be present (see Figure 2 and Table 4). The N II, Si II, and Si III lines follow the narrow H I absorption, as does the blue component of the $\mathrm{C}$ III line; the redward $\mathrm{C}$ III line and the $\mathrm{N}$ III line match the location of the BLA and O VI. The narrower blue component likely has a low temperature consistent with PIE, and is closely associated with a nearby $2 L^{*}$ galaxy $168 \mathrm{kpc}\left(0.8 R_{\mathrm{vir}}\right)$ away. Using the measured $b$-values for the BLA and $\mathrm{OVI}$ in this case suggests a gas temperature in CIE of $T \approx 8 \times 10^{5} \mathrm{~K}$. This probable BLA may be associated either with the nearby galaxy or the entire galaxy group, since the velocity difference between the broad and 
narrow components suggests different physical locations for these absorbers.

Group 32123. In this sight line, there are two possibilities for BLAs. One at $z=0.16081$ contains broad O VI absorption seen in both lines of the doublet but at low-S/N. A BLA corresponding to this $\mathrm{O}$ VI can be fit within the Ly $\alpha$ absorption complex, but is not well constrained - that is, we can set only a very loose upper limit on temperature $\left(T<8 \times 10^{5} \mathrm{~K}\right)$ by assuming that the observed O VI line width is entirely thermal. More interesting is a possible, metal-free BLA absorber at $z=0.16142$, whose symmetrical line width suggests a temperature of $1.5 \times 10^{5} \mathrm{~K}$, which would make it a collisionally ionized absorber. However, the limited $\mathrm{S} / \mathrm{N}$ of this weak feature means that it can also be a blend of two narrower components.

Group 36001. In this spectrum at $z=0.18527$, there is a weak, broad O VI $1032 \AA$ absorber-too weak to be detected in the $1038 \AA$ line. While a BLA could be present, its parameters are not well constrained. As with the possible warm absorber in group 32123, we set a very loose upper limit of $T<1 \times 10^{6} \mathrm{~K}$ by assuming that the OVI line width is entirely thermal. Two possibilities seem equally plausible: a warm absorber with a BLA and broad O VI and a cool absorber with narrow $\mathrm{H} \mathrm{I}$ and Si III are both present, or there is a single cool absorber that contains both photoionized $\mathrm{Si}$ III and OVI. There is also another $\mathrm{HI}$ plus $\mathrm{OVI}$ absorber possibly associated with group 36001 at a relative velocity of $-906 \mathrm{~km} \mathrm{~s}^{-1},>2.5 \sigma_{\text {grp }}$ from the group mean velocity and thus outside the velocity bounds we have set for association (see Section 5.1). In this case, the HI and OVI have nearly the same line widths, suggesting a low temperature and a turbulent medium, but a BLA associated with the O VI could be present in the saturated Ly $\alpha$ profile. Assuming that the O VI width is entirely thermal sets a firm upper limit of $T<1 \times 10^{6} \mathrm{~K}$.

Group 44739. In this case, a very broad, shallow O VI $1032 \AA$ line is well-aligned with a narrow $\mathrm{H} \mathrm{I}$ line that also has moderately strong Si III (see Figure 1, online panel 9 and Table 10) at $z=0.11766$. While a BLA could be present in this case, and is suggested by the breadth of the symmetrical O VI doublet lines, the BLA is predicted to have $b_{\mathrm{HI}} \approx 200 \mathrm{~km} \mathrm{~s}^{-1}$, assuming minimal gas turbulence or velocity components in the $\mathrm{O}$ VI. This line width is so broad that the predicted BLA would be undetectable, and suggests $T>10^{6} \mathrm{~K}$. In this scenario, a cool, photoionized absorber would also be present at nearly the same velocity, based on the narrow $\mathrm{HI}$ and Si III. But the presence of this BLA is very uncertain.

Group 44858. In this case, the original line fit listed in Table 10 for this $z=0.12567$ absorber does not change by applying the method used previously. The somewhat broad H I and $\mathrm{O}$ VI lines indicate a gas temperature just slightly in excess of $10^{5} \mathrm{~K}$; however, given the uncertain amount of non-thermal motion contributing to the line widths, we cannot prove that this absorber is collisionally ionized warm gas. The nearest galaxy is $7 R_{\text {vir }}$ away, making a group association with this gas most likely.

In summary, there are two probable BLAs in this sample of absorbers, one of which is possibly associated with an individual galaxy $(25124 / 0.18540)$. One other system (44858/0.12567), with H I and O VI only, has line widths close to but not definitively within the collisionally ionized regime. Additionally, there are four possible BLAs, including three systems with H I, O VI, and Si III (with line width-inferred temperature limits in excess of $10^{5} \mathrm{~K}$ ), and one $\mathrm{H}$ I-only BLA $(32123 / 0.16142)$ with a broad, symmetrical profile yielding a temperature estimate of $1.5 \times 10^{5} \mathrm{~K}$, assuming the line width is entirely thermal. One other system $(36001 / 0.18429)$ has a poorly measured upper limit on temperature of $<10^{6} \mathrm{~K}$, but is outside the velocity bounds established for association with its group. A separate absorber in this same group (36001/ 0.18527 ) has a possible BLA that sets a very similar limit on temperature.

Overall, this survey detected eight absorbers in six groups with metal lines consistent with being in PIE at a fiducial temperature of $\sim 2 \times 10^{4} \mathrm{~K}$. Two probable BLA absorbers have temperature estimates at $T \gtrsim 10^{5} \mathrm{~K}$, and four other possible BLAs are present with temperature estimates or limits in the collisionally ionized regime. However, unlike in Paper II there is no strong evidence for warm gas ubiquitously associated with the galaxy groups in this sample.

\section{Characterizing the SDSS Groups Probed by These Sight Lines}

\subsection{MOS Observations}

While the acquisition and analysis of the galaxy redshift and magnitude data is presented and described in detail in Keeney et al. (2018), here we summarize the basic parameters of those observations as they apply to the group membership analysis. For all of the target sight line fields, the SDSS spectroscopic survey data is used for the brighter galaxies $(r \leqslant 17.8)$, while new MOS on fainter galaxies was obtained specifically for this program at the MMT Observatory $6 \mathrm{~m}$ using the Hectospec MOS (Fabricant et al. 2005) and at the WIYN $3.5 \mathrm{~m}$ telescope using the HYDRA MOS.

These groups have modest numbers of SDSS galaxies $\left(N_{0}=3-10\right)$, so we designed the MOS survey to increase the number of group members to $N \geqslant 20$ per group to better constrain basic group parameters, including velocity dispersion, centroid position, and extent on the sky, which can be used to infer, for example, the group halo mass and group virial radius. Our goal for these observations was not very high completeness (i.e., $>90 \%$ ), but rather a fair sampling of potential group members so that the group is well-characterized. The overall completeness of these observations is $\geqslant 60 \%$ for these target fields, where the overall completeness is defined to be the fraction of all targets with $g<20$ within $20^{\prime}$ of the sight line for which redshifts are available from this survey or other sources (Keeney et al. 2018, see their Table 8 for individual field completeness values and other related quantities). These limits correspond to group galaxies at $L \geqslant 0.2 L^{*}$, although only a small percentage of those galaxies with redshifts in-hand are actually at the redshifts of these groups; it is likely that a similarly small percentage of unobserved galaxies are also at the group redshift.

The targeting of galaxies in these fields was blind with respect to galaxy color, type, or clustering, but rather relied entirely on the apparent magnitude, proximity to the sight line and SDSS photometric redshift estimate (galaxies with $z_{\text {phot }}>0.25$ were targeted at lower priority). See Keeney et al. (2018) for more details on the targeting process. The brightest galaxies $\left(g<18\right.$ or $L \geqslant L^{*}$ at the redshifts of these groups) and the closest galaxies $\left(\rho<5^{\prime}\right.$ or $\approx 700 \mathrm{kpc}$ at the redshifts of these groups) were targeted with highest priority. This results in completeness fractions of $\gtrsim 75 \%$ for the closest 
galaxies down to very faint magnitudes $\left(g<20\right.$ or $L \gtrsim 0.2 L^{*}$ at the group redshifts) and for the brightest galaxies ( $g \leqslant 18$ or $\sim L^{*}$ at the group redshifts) out to $\sim 2.5 \mathrm{Mpc}$ from the sight lines (see Appendix A in Keeney et al. 2018 for statistics by sight line). These completeness fractions are for all galaxies with these parameters, not just the group galaxies. The high completeness fractions down to faint limits close to these sight lines means that it is quite unlikely that we have missed a nearest galaxy to these absorbers (see the next subsection). The high completeness for bright galaxies out to large radii means that the full extent and constituency of these groups has been very well sampled.

At WIYN/HYDRA, we used the 600@10.1 grating centered at $5200 \AA(\mathcal{R} \approx 1200$ from $3800-6600 \AA)$; the $\mathrm{S} / \mathrm{N}$ of these spectra is $9_{-3}^{+5}$ per pixel when the galaxy $g$-band magnitude range is $19.4_{-0.7}^{+0.5}$. At MMT/Hectospec, we used the $270 \mathrm{gpm}$ grating to achieve $\mathcal{R} \approx 1000$ from 3700 to $9100 \AA$; the $\mathrm{S} / \mathrm{N}$ of these spectra is $7_{-3}^{+6}$ per pixel when the galaxy $g$-band magnitude range is $19.8_{-0.8}^{+0.7}$. We chose our wavelength coverage to ensure good sensitivity to $\mathrm{Ca}$ II $\mathrm{H} \& \mathrm{~K}$ absorption at $z \approx 0$ to maximize the return of accurate redshifts for absorption-line galaxies in these low- $z$ galaxy groups.

The design criterion to obtain redshifts for a representative sample of $>20$ group members is achieved in all but two cases, group 36001 (6 group galaxies; see Table 8) and group 44726 (8 group members; Table 8). Group 36001 has only $57 \%$ overall completeness, but Group 44726 easily exceeds the design completeness level $(60 \%)$ for this survey. Since the MOS on these two fields did not differ significantly in completeness from the others, these are truly sparse groups, not fields with high incompleteness.

\subsection{Nearest Galaxies to the Absorbers}

For all of our groups, we have located the galaxy with $\left|\Delta v_{\text {abs }}\right| \leqslant 1000 \mathrm{~km} \mathrm{~s}^{-1}$ nearest to the QSO sight line to determine whether an individual galaxy is more likely associated with the observed absorption than the group as a whole. While our original criteria for choosing sight lines excluded QSOs whose impact parameter to a bright group galaxy was $\leqslant 1.5 R_{\text {vir }}$, deeper MOS found two cases where fainter galaxies were found close to the sight line at the redshift of the absorber.

Table 5 lists the properties of the nearest galaxy to each absorber detected in our $H S T / C O S$ spectra at or near the group redshift. Column 9 lists the rest-frame velocity difference between the absorber and galaxy (i.e., $\left.\Delta v_{\mathrm{abs}}=c\left(z_{\mathrm{gal}}-z_{\mathrm{abs}}\right) /\left(1+z_{\mathrm{abs}}\right)\right)$. The final column lists the galaxy's three-dimensional distance from the absorber, $D$, normalized by the galaxy's virial radius, $R_{\text {vir }}$. The virial radius is estimated from the galaxy's rest-frame $g$-band luminosity, $L_{\text {gal }}{ }^{4}$ as detailed in Stocke et al. (2013). The three-dimensional absorber-galaxy distance is calculated using

$$
D^{2}=\rho^{2}+D_{z}^{2}
$$

where $\rho$ is the galaxy's impact parameter with respect to the QSO sight line and $D_{z}$ is the distance along the line of sight in Mpc. The line-of-sight distance is calculated using a reduced

\footnotetext{
4 As in Keeney et al. (2017), we adopt $M_{g}^{*}=-20.3$ (Montero-Dorta \& Prada 2009, corrected to $H_{0}=70 \mathrm{~km} \mathrm{~s}^{-1}$ ) with $K$-corrections from Chilingarian et al. (2010) and Chilingarian and Zolotukhin (2012).
}

Hubble flow model such that

$$
D_{z}= \begin{cases}0 & \left|\Delta v_{\mathrm{abs}}\right| \leqslant v_{\text {red }} \\ \left(\left|\Delta v_{\mathrm{abs}}\right|-v_{\mathrm{red}}\right) / H_{0} & \left|\Delta v_{\mathrm{abs}}\right|>v_{\text {red }}\end{cases}
$$

We assume $v_{\text {red }}=400 \mathrm{~km} \mathrm{~s}^{-1}$ for consistency with previous studies of galaxy-absorber associations (see, e.g., Paper II, Prochaska et al. 2011; Stocke et al. 2013; Keeney et al. 2017, 2018; Pratt et al. 2018). This convention is used to account for possible peculiar velocities between galaxies and absorbers (e.g., due to outflow/infall); the reduced Hubble flow cutoff value was chosen to match the maximum rotation speeds of the most massive galaxies.

There are only two cases for which a galaxy is close to the sight line in units of virial radii: group $25124\left(0.8 R_{\mathrm{vir}}\right.$ from the sight line) and group $32123\left(0.6 R_{\text {vir }}\right.$ away). Based on the observed distributions of nearest and next-nearest neighbors to a large sample of absorbers, Keeney et al. (2017) find that low$z$ absorption can be attributed with confidence to the CGM of individual galaxies only when $D / R_{\text {vir }} \lesssim 1.4$. This suggests that some of the absorption in groups 25124 and 32123 is likely associated with individual galaxies in Table 5 rather than the group itself.

To investigate this uncertainty, the sight line toward FBQS 1010+3003 was used as a test case for the current study. This sight line was selected in the same way as the current study, except that a bright SDSS galaxy at the group redshift was known to be present close to the sight line prior to deeper MOS. A relatively high-S/N (15-20) COS spectrum was in-hand for FBQS $1010+3003$ from the science program of the COS-GTOs (Stocke et al. 2013; Keeney et al. 2017), and a deep galaxy survey of the region is available from Keeney et al. (2018). A detailed examination in Stocke et al. (2017) tried to distinguish between an absorber association with an individual $2.7 L^{*}$ galaxy located $1.0 R_{\text {vir }}$ from the absorber and a small group of nearby galaxies, of which the individual galaxy is not a member. For an O VI-only absorber in the FUV spectrum of FBQS $1010+3003$, a better match both in sky position and velocity was found for the individual galaxy association. For comparison, the nearest galaxy data for this case is reproduced at the bottom of Table 5 and labeled group 49980. The O VI-only absorber is at $z=0.11351$; the other absorbers are detected in $\operatorname{Ly} \alpha$, some of which likely are associated with the galaxy group (see Stocke et al. 2017 for details).

The situation is not so clear for groups 25124 and 32123. For the $z=0.18529,0.18540$ (the BLA plus O VI absorber), 0.18569 Ly $\alpha$ complex in group 25124, the high and low-ion components cannot be at the same location, given their velocity difference. All three components can be associated with the single galaxy in Table 5, or the BLA plus broad O VI can be associated with the galaxy group. To be conservative, we associate all three with the galaxy and count group 25124 as a non-detection. The case of group 32123 is somewhat clearer in that a total of four absorbers are present; the two at lower velocity match the velocity of the nearest galaxy (see Table 5), which is not a group member. The other two absorbers are too far away in velocity to be plausibly associated with this same galaxy. For these two absorbers, which include an H I and O VI absorber and a BLA (see Figure 1), the closest galaxy is $1.8 R_{\text {vir }}$ away, so we identify the absorption with the galaxy group. 
Table 5

Nearest Galaxy Properties

\begin{tabular}{|c|c|c|c|c|c|c|c|c|c|}
\hline (1) & $\begin{array}{l}z_{\text {abs }} \\
\text { (2) }\end{array}$ & $\begin{array}{l}\text { R.A. } \\
\left({ }^{\circ}\right) \\
(3)\end{array}$ & $\begin{array}{c}\text { Decl. } \\
\left({ }^{\circ}\right) \\
(4)\end{array}$ & $\begin{array}{l}z_{\mathrm{gal}} \\
(5)\end{array}$ & $\begin{array}{c}L_{\mathrm{gal}} \\
\left(L^{*}\right) \\
(6)\end{array}$ & $\begin{array}{c}R_{\mathrm{vir}} \\
(\mathrm{kpc}) \\
(7)\end{array}$ & $\begin{array}{c}\rho \\
(\mathrm{kpc}) \\
(8)\end{array}$ & $\begin{array}{c}\Delta v_{\text {abs }} \\
\left(\mathrm{km} \mathrm{s}^{-1}\right) \\
(9)\end{array}$ & $\begin{array}{c}D / R_{\text {vir }} \\
(10)\end{array}$ \\
\hline 12833 & 0.14743 & 129.343638 & 44.390149 & 0.14844 & 2.6 & 248 & 772 & 263 & 3.1 \\
\hline 16803 & 0.14497 & 235.046611 & -2.039641 & 0.14549 & 1.2 & 191 & 525 & 136 & 2.7 \\
\hline 19670 & 0.13388 & 149.958823 & 50.778650 & 0.13346 & 1.1 & 185 & 499 & -111 & 2.7 \\
\hline 25124 & $\begin{array}{l}0.18529 \\
0.18569\end{array}$ & 243.531018 & 26.556435 & 0.18507 & 1.9 & 223 & 168 & $\begin{array}{r}-55 \\
-159\end{array}$ & 0.8 \\
\hline 32123 & $\begin{array}{l}0.15874 \\
0.16081 \\
0.16142\end{array}$ & $\begin{array}{l}203.271113 \\
203.211036\end{array}$ & $\begin{array}{l}45.315150 \\
45.300789\end{array}$ & $\begin{array}{l}0.15853 \\
0.16102\end{array}$ & $\begin{array}{l}5.0 \\
0.78\end{array}$ & $\begin{array}{l}311 \\
167\end{array}$ & $\begin{array}{l}175 \\
299\end{array}$ & $\begin{array}{r}-54 \\
54 \\
-103\end{array}$ & $\begin{array}{l}0.6 \\
1.8\end{array}$ \\
\hline 36001 & $\begin{array}{l}0.18429 \\
0.18494 \\
0.18527\end{array}$ & $\begin{array}{l}156.986890 \\
156.986060\end{array}$ & $\begin{array}{l}21.245411 \\
21.369490\end{array}$ & $\begin{array}{l}0.18432 \\
0.18658\end{array}$ & $\begin{array}{l}2.7 \\
2.0\end{array}$ & $\begin{array}{l}252 \\
228\end{array}$ & $\begin{array}{r}1239 \\
887\end{array}$ & $\begin{array}{r}7 \\
414 \\
-323\end{array}$ & $\begin{array}{l}4.9 \\
4.0 \\
3.9\end{array}$ \\
\hline & 0.18585 & 157.086777 & 21.355513 & 0.18752 & 4.7 & 304 & 382 & 422 & 1.6 \\
\hline $\begin{array}{l}44564 / 44565 \\
44726\end{array}$ & $\begin{array}{l}\ldots \\
\ldots\end{array}$ & $\begin{array}{l}\ldots \\
\ldots\end{array}$ & $\begin{array}{l}\ldots \\
\ldots\end{array}$ & $\begin{array}{l}\ldots \\
\ldots\end{array}$ & $\begin{array}{l}\cdots \\
\ldots\end{array}$ & $\begin{array}{l}\cdots \\
\ldots\end{array}$ & $\begin{array}{l}\cdots \\
\ldots\end{array}$ & $\begin{array}{l}\cdots \\
\ldots\end{array}$ & $\begin{array}{l}\cdots \\
\ldots\end{array}$ \\
\hline 44739 & $\begin{array}{l}0.11537 \\
0.11706 \\
0.11766 \\
0.11795\end{array}$ & $\begin{array}{l}229.950843 \\
229.844996\end{array}$ & $\begin{array}{l}28.626721 \\
28.711684\end{array}$ & $\begin{array}{l}0.11502 \\
0.11801\end{array}$ & $\begin{array}{l}1.1 \\
1.4\end{array}$ & $\begin{array}{l}185 \\
203\end{array}$ & $\begin{array}{l}350 \\
661\end{array}$ & $\begin{array}{r}-94 \\
254 \\
93 \\
16\end{array}$ & $\begin{array}{l}1.9 \\
3.3\end{array}$ \\
\hline 44858 & $\begin{array}{l}0.12551 \\
0.12567\end{array}$ & 230.069021 & 28.838277 & 0.12638 & 4.0 & 287 & 2006 & $\begin{array}{l}231 \\
189\end{array}$ & 7.0 \\
\hline 50433 & $\ldots$ & $\cdots$ & $\cdots$ & $\cdots$ & $\cdots$ & $\cdots$ & $\cdots$ & $\cdots$ & $\cdots$ \\
\hline 49980 & $\begin{array}{l}0.11351 \\
0.11381 \\
0.11466 \\
0.11675\end{array}$ & 152.472930 & 30.033939 & 0.11345 & 2.7 & 252 & 254 & $\begin{array}{r}-16 \\
-96 \\
-325 \\
-332\end{array}$ & 1.0 \\
\hline
\end{tabular}

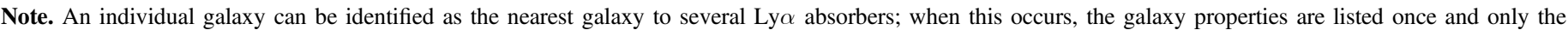

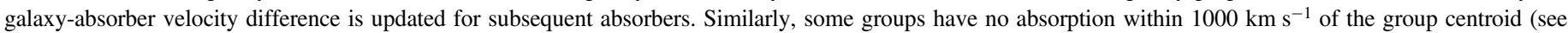
Table 10), as indicated by the "..." symbol in each column.

The individual galaxies associated with absorbers near the redshifts of groups 25124 and 32123 are both early types, with specific star formation rates consistent with being passive galaxies (sSFR $<10^{11} \mathrm{yr}^{1}$ ). Additionally, the nearest galaxy in the FBQS $1010+3003$ sight line is an Sa galaxy whose sSFR suggests that it is also passive. We detect $\mathrm{O}$ VI absorption in all three of these cases, as well as a few other cases in previous investigations (Paper II and Keeney et al. 2017), but the COSHalos collaboration found very few O VI absorptions associated with passive galaxies in their sample (Tumlinson et al. 2011). The reported absence of $\mathrm{OVI}$ is almost certainly due to the COS-Halos spectra having lower $\mathrm{S} / \mathrm{N}$ than the present data and the data obtained in the COS-GTO program, resulting in a rather high column density $\operatorname{limit}\left(\log N_{\mathrm{O} \text { VI }}>14.3\right)$ for their detections. While O VI is undoubtedly present around most passive galaxies, the larger impact parameters of those absorbers suggests an association with the entire group to which the galaxy is a member (Paper II and Keeney et al. 2017). The rather constant covering fraction of $50 \%-70 \%$ for absorption-line galaxies as a function of impact parameter out to several virial radii $\left(4 R_{\mathrm{vir}}\right.$ or $\left.\sim 1 \mathrm{Mpc}\right)$ suggests that the gaseous structure creating the absorption around passive galaxies is much larger than an individual galaxy CGM (see Figures 7 and 8 in Keeney et al. 2018).

For the other groups, despite the much deeper MOS obtained by us, there are no galaxies close to the sight lines, making the claim that these absorbers are associated with the entire galaxy group strong. The full range of nearest galaxy impact parameters is shown as a histogram in Figure 3. The bins are in units of virial radii, and open symbols indicate $\mathrm{HI}$-only absorption, red indicates low-ion without $\mathrm{OVI}$, and blue indicates O VI absorption with or without low ions. The FBQS $1010+3003$ field listed at the bottom as group 49980 is included for completeness, despite having a previously known galaxy close to the sight line (see Stocke et al. 2017).

In summary, while all the absorptions listed in Table 5 could be ascribed to the foreground groups targeted by this study, we conservatively associate the absorption complex in group 25124 and two of four absorbers near group 32123 to the individual closest galaxy listed in Table 5. We associate the other two absorbers near group 32123 (those not close to an individual galaxy) with the entire group. That is, we count group 25124 as a non-detection, while group 32123 is counted as a detection because only one of the two absorber systems detected is associated with an individual galaxy.

\subsection{Group-finding Algorithm and Parameters}

The group-finding algorithm employed here differs somewhat from that presented in our previous group studies. Paper II and Stocke et al. (2017) used a friends-of-friends (FoF) algorithm to create an initial guess at group membership, which was subsequently refined by a "virialization" step. Here, we remove the initial FoF step altogether and use Monte Carlo 


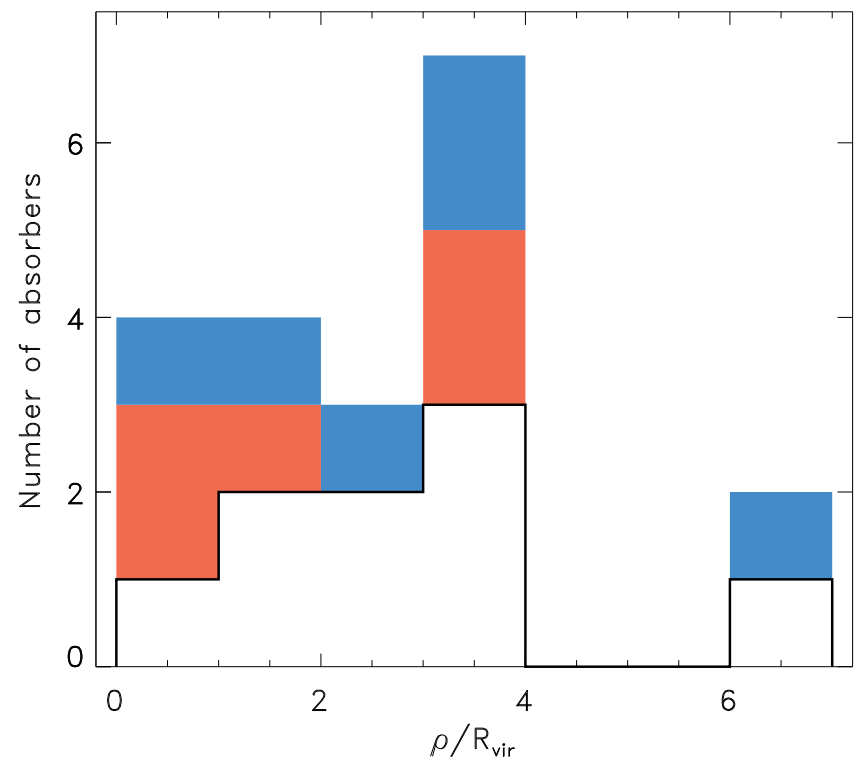

Figure 3. Impact parameters of nearest galaxies to the absorbers in this survey. Open boxes are metal-free absorbers; blue boxes indicate the presence of $\mathrm{O} \mathrm{VI}$, while the red boxes are absorbers with only low ions. The FBQS $1010+3003$ field, which has a close galaxy at $\rho / R_{\text {vir }}<1$, is included in this compilation despite being chosen somewhat differently from the rest of the sample (see text).

simulations to explore the systematic effects of initial parameters in the virialization procedure.

Our group-finding algorithm assumes a group mass-to-light ratio of $\Upsilon_{\text {grp }} \equiv M_{\text {grp }} / L_{\text {grp }}$, which allows us to estimate the group's virial radius, $R_{\text {grp }}$, and velocity dispersion, $\sigma_{\text {grp }}$, based on the total luminosity of the group members:

$$
\begin{gathered}
L_{\text {grp }}=\sum_{i=1}^{N_{\text {grp }}} L_{i}, \\
M_{\text {grp }}=\Upsilon_{\text {grp }}\left(\frac{L_{\text {grp }}}{L^{*}}\right) \times 10^{10} M_{\odot}, \\
R_{\text {grp }}=\left(\frac{3 M_{\text {grp }}}{4 \pi \Delta_{\text {crit }} \rho_{\text {crit }}}\right)^{1 / 3} \\
=957\left(\frac{M_{\text {grp }}}{10^{14} M_{\odot}}\right)^{1 / 3} \mathrm{kpc}, \\
\sigma_{\text {grp }}=\sqrt{\frac{G M_{\text {grp }}}{3 R_{\text {grp }}}} \\
=387\left(\frac{M_{\text {grp }}}{10^{14} M_{\odot}}\right)^{1 / 3} \mathrm{~km} \mathrm{~s}^{-1} .
\end{gathered}
$$

We assume $\Delta_{\text {crit }}=200$ and $\rho_{\text {crit }}=9.205 \times 10^{-30} h_{70}^{2} \mathrm{~g} \mathrm{~cm}^{-3}$ (Shull et al. 2012), and ignore any evolution with redshift since our groups are all located at $z<0.2$.

We start with an initial group position, redshift, and mass close to those in Table 2, then search for galaxies that are located within a specified distance of the group center on the plane of the sky and in velocity space (see Section 4.3.1 for details). All galaxies in this volume are assumed to be group members, and new group positions, redshifts, and masses are estimated based on the revised membership. These updated group properties are used as the starting point for the next iteration of the group finder, and the algorithm iterates until it converges (i.e., the identities of group members do not change from one iteration to the next), which is typically achieved in five or fewer iterations.

As mentioned previously, all galaxies within our search volume are considered to be group members, so it is important to choose the boundaries carefully. For the boundary on the plane of the sky, we adopt the group splashback radius, $R_{\mathrm{sp}} \sim \Omega_{\mathrm{m}}^{-1 / 3} R_{\mathrm{grp}} \approx 1.5 R_{\mathrm{grp}}$ (Diemer 2017). For the boundary in velocity space, we adopt $\pm 2 \sigma_{\text {grp }}$ from the group redshift. This value was chosen by analyzing a large mock catalog, where galaxies were placed inside dark matter halos in an $N$-body simulation and given velocities that trace the velocities of dark matter particles; this analysis suggests that $>99 \%$ of all satellite galaxies are located within $2 \sigma_{\operatorname{grp}}$ of the parent halo's velocity.

\subsubsection{Monte Carlo Analysis}

The basic group properties that we wish to derive are its position on the sky, redshift, and membership. Unfortunately, these properties depend sensitively on nuisance parameters ${ }^{6}$ whose values are unknown or ill-constrained. We employ Monte Carlo simulations to marginalize over these parameters by allowing them to vary over a range of values.

Our initial estimates of the group location, redshift, and mass come from the SDSS analysis in Table 2. The uncertainties in the group position and redshift are the uncertainties in the mean value for the SDSS group members (i.e., they are proportional to $N_{0}^{-1 / 2}$ ). We assume Gaussian priors for these values, with mean and standard deviation from Table 2.

The uncertainties in the group mass were estimated by running the SDSS FoF algorithm of Paper III on mock galaxy catalogs where the true halo masses were known. Our prior on $\log M_{0}$ assumes a Gaussian with mean and standard deviation listed in Table 2 that is then weighted by a halo mass function (HMF) to account for the expectation that low-mass halos are more common than high-mass halos. The HMF was evaluated at $z=0.15$ with $\Delta_{\text {crit }}=200$, cosmological parameters from Planck (Planck Collaboration et al. 2014), and fitting functions from Tinker et al. (2008) using the online HMF calculator of Murray et al. (2013).

We adopt a Gaussian prior on $\log \Upsilon_{\text {grp }}$ with a mean of 2.5 and a standard deviation of 0.3 dex, which is a good characterization of the $g$-band values from Proctor et al. (2015). While it is typically assumed that redder optical magnitudes (i.e., SDSS $r$ - or $i$-band) correlate more strongly with an individual galaxy's stellar mass, $\Upsilon_{\text {grp }}$ shows no difference in correlation for the SDSS $g$-, $r$-, and $i$-bands (i.e., the Gaussian distribution of $\log \Upsilon_{\text {grp }}$ has a width of 0.3 dex in all three bands; Proctor et al. 2015). Thus, we retain the $g$-band as our standard of reference for all galaxy luminosities for consistency with past studies (Paper II, Stocke et al. 2013, 2017; Keeney et al. 2017, 2018).

For each SDSS galaxy group in Table 2, we perform 10,000 Monte Carlo realizations, allowing the nuisance parameters to vary as described previously. For each realization, we record

\footnotetext{
5 The "splashback radius" is the apocenter of satellites during their first orbit after falling into a dark matter halo, and has been suggested as a physically motivated definition of a halo boundary (Adhikari et al. 2014; Diemer \& Kravtsov 2014; More et al. 2015).

6 We use the term "nuisance parameter" in the statistical sense; that is, a model parameter that is not of physical interest but must be accounted for to constrain parameters that are physically interesting.
} 
the values of the nuisance parameters for that trial and the group members identified by our algorithm. From these, physically meaningful values such as the group position, redshift, and luminosity, $M_{\text {grp }}, R_{\text {grp }}, \sigma_{\text {grp }}$, are derived. ${ }^{7}$ The observed size $\left(R_{\mathrm{obs}}\right)$ and velocity dispersion $\left(\sigma_{\mathrm{obs}}\right)$ of the group members, and the impact parameter between the group center and the QSO sight line $\left(\rho_{\star}\right)$, also are derived. The ensemble of all realizations are then analyzed to determine the range of the derived quantities for each galaxy group.

As in Paper II and Stocke et al. (2017), we have used robust estimators of the group properties, as initially defined by Beers et al. (1990). The position of the group on the sky and its redshift are calculated with the bi-weight location estimator, and the observed velocity dispersion of the group $\left(\sigma_{\text {obs }}\right)$ is calculated using the "gapper" scale estimator, as illustrated in Equations (3)-(4) of Paper II. Additionally, we estimate the observed size of the group $\left(R_{\text {obs }}\right)$ by calculating each group member's impact parameter with respect to the group center and adopting the 68 th percentile value as $R_{\text {obs }}$ (i.e., $68 \%$ of the identified group members are within a projected distance of $R_{\text {obs }}$ from the group center). Unlike $\sigma_{\text {obs }}$, we do not assign any physical meaning to $R_{\text {obs}}$; it is merely an empirical means of assessing whether the identified group is approximately the size that we would expect given its inferred mass (i.e., $R_{\text {obs }} \sim R_{\text {grp }}$ ).

Table 6 summarizes our Monte Carlo realizations. Column 1 lists the group identifier, columns 2 and 3 the sky position in degrees, and column 4 the redshift $\left(z_{\text {grp }}\right)$. Column 5 lists the number of group members $\left(N_{\text {grp }}\right)$, column 6 the total group luminosity ( $L_{\mathrm{grp}}$; Equation (3)), and column 7 the total group mass $\left(M_{\text {grp }}\right.$; Equation (4)). Columns 8 and 9 are the observed group radius $\left(R_{\text {obs }}\right)$ and velocity dispersion $\left(\sigma_{\text {obs }}\right)$. Column 11 lists the ratio $R_{\mathrm{obs}} / R_{\mathrm{grp}}$, and column 12 the ratio $\sigma_{\mathrm{obs}} / \sigma_{\text {grp }}$, where $R_{\text {grp }}$ and $\sigma_{\text {grp }}$ are calculated using Equations (5) and (6), respectively. Columns 10 and 13 list the sight line impact parameter from the group centroid in $\mathrm{kpc}$ and as a fraction of $R_{\text {grp }}$. Details of individual groups are discussed in Appendix B.

\subsection{Adopted Group Membership}

The Monte Carlo analysis described in Section 4.3 allows us to assess which galaxies are group members probabilistically. Regardless of the uncertainties of this process, it is essential that a final group membership is adopted so that its overall properties (mean recession velocity, mass, etc.) and relationship to any absorption can be assessed, as well as whether the nearest galaxy to the QSO sight line is a group member (e.g., the passive galaxy closest to one of the absorbers in Group 32123 is not a member of the group identified in this field; Stocke et al. 2017).

As a first step, we determine each galaxy's membership fraction, $f_{\text {mem }}$ (i.e., the fraction of the Monte Carlo trials in which a particular galaxy was identified as a group member). While it is tempting to define the final group as the set of all galaxies with $f_{\text {mem }}>50 \%$, there is no guarantee that this set of galaxies is self-consistent (i.e., all located within $1.5 R_{\text {grp }}$ of the group center and $2 \sigma_{\text {grp }}$ of the group redshift, to the exclusion of all other galaxies).

\footnotetext{
We caution the reader that we are using different notation than Paper II and Stocke et al. (2017). We now call the group velocity dispersion expected based on its mass $\sigma_{\text {grp }}$ instead of $\sigma_{\text {vir }}$, and the observed velocity dispersion $\sigma_{\text {obs }}$ instead of $\sigma_{\text {grp }}$. We believe that this new nomenclature is more transparent and internally consistent, and apologize for any confusion.
}

Instead, we calculate several group parameters (sky position, $z_{\mathrm{grp}}, N_{\mathrm{grp}}, M_{\mathrm{grp}}$ ) for each Monte Carlo realization, and compare them with the distribution of values over all realizations (i.e., marginal likelihoods ${ }^{8}$ ) to determine whether the value for a particular realization is a common outcome. This comparison uses an adaptive kernel density estimate (Terrell \& Scott 1992) of the marginal likelihoods to avoid artifacts from arbitrary choices of histogram bin sizes and centers.

Each realization is then assigned a joint likelihood by multiplying the marginal likelihood values described previously over all parameters. We adopt the membership of the realization with the highest joint likelihood as the final group configuration. This procedure ensures that we only adopt group configurations that are representative and self-consistent.

Figure 4 shows the adopted membership of individual groups, with group members displayed in color and the gradation in color indicating the recession velocity gradient for group members. Gray symbols are non-group galaxies in the field-of-view and velocity range. Zero velocity in the top histogram and the center in the bottom plot are the SDSS group velocity and sky position in Table 2, respectively. The solid vertical line in the top panel, and the " $\times$ " symbol in the bottom panel, mark the adopted group velocity and sky centroids, respectively. The star marks the AGN sight line location in the bottom panel, while the absorber velocity or velocities are shown as dashed vertical lines in the top panel.

Table 7 lists the adopted members of individual galaxy groups, with galaxies listed in order of decreasing membership fraction, $f_{\text {mem. }}$. This table includes by column (1) a galaxy identifier, which is either a SDSS "ObjID" or, for galaxies without SDSS redshifts, a string in the style of Keeney et al. (2018); (2 and 3) galaxy sky position in degrees; (4) galaxy redshift; (5) rest-frame $g$-band luminosity; (6) galaxy spectral type; (7 and 8) galaxy offset on the sky (in kpc) and in velocity (in $\mathrm{km} \mathrm{s}^{-1}$ ) from the SDSS group centroids in Table 2; and (9) $f_{\text {mem. }}$ A galaxy's spectral type is determined visually as described in Keeney et al. (2018), and abbreviated "E" for emission-line galaxies, "A" for absorption-line galaxies, and " $\mathrm{C}$ " for composite galaxies that show both emission and absorption.

The properties of our adopted groups are summarized in Table 8 , where all values are calculated using only galaxies that are adopted as group members by the procedure described previously. The columns list largely the same information as those of Table 6, except for columns 12 and 13, which list the offsets between the adopted sky position and redshift and the SDSS values (Table 2), and column 14, which lists the group's spiral fraction. We estimate a group's spiral fraction using the spectral types of the members, where all emission-line and composite galaxies are considered "spirals." These percentages use only those galaxies at $L>0.6 L^{*}$ where the MMTO galaxy survey work is largely complete (Keeney et al. 2018).

The centroids for 9 of the 12 groups did not vary significantly between the SDSS analysis and the results found here using much deeper data. The deeper MOS data find that groups 44564 and 44565 in the CSO 1022 sight line are part of a larger group with centroids intermediate between the two, and the new analysis finds group 12833 in the RBS 711 sight line to be $1.5 \sigma\left(\sim 300 \mathrm{~km} \mathrm{~s}^{-1}\right)$ lower in redshift than in Table 2 . The remaining 9 groups showed little change in their 3D locations between Tables 2 and 8. Derived halo masses range from $10^{13.5}$ to $10^{14.6} M_{\odot}$, with a corresponding

\footnotetext{
These are the histograms in Figure 15 (see Appendix B)
} 
Table 6

Range of Group Properties

\begin{tabular}{|c|c|c|c|c|c|c|c|c|c|c|c|c|}
\hline Group & $\begin{array}{l}\text { R.A. } \\
\left({ }^{\circ}\right) \\
(2)\end{array}$ & $\begin{array}{c}\text { Decl. } \\
\left({ }^{\circ}\right) \\
(3)\end{array}$ & $\begin{array}{l}z_{\text {grp }} \\
(4)\end{array}$ & $\begin{array}{r}N_{\text {grp }} \\
(5)\end{array}$ & $\begin{array}{l}L_{\mathrm{grp}} \\
\left(L^{*}\right) \\
(6)\end{array}$ & $\begin{array}{c}\log M_{\text {grp }} \\
\text { (7) }\end{array}$ & $\begin{array}{c}R_{\mathrm{obs}} \\
(\mathrm{kpc}) \\
(8)\end{array}$ & $\begin{array}{c}\sigma_{\mathrm{obs}} \\
\left(\mathrm{km} \mathrm{s}^{-1}\right) \\
(9)\end{array}$ & $\begin{array}{c}\rho_{\star} \\
(\mathrm{kpc}) \\
(10)\end{array}$ & $\begin{array}{c}R_{\mathrm{obs}} / R_{\mathrm{grp}} \\
(11)\end{array}$ & $\begin{array}{c}\sigma_{\mathrm{obs}} / \sigma_{\mathrm{grp}} \\
(12)\end{array}$ & $\begin{array}{c}\rho_{\star} / R_{\text {grp }} \\
(13)\end{array}$ \\
\hline 12833 & $129.400_{-0.013}^{+0.013}$ & $44.282_{-0.007}^{+0.010}$ & $0.14584_{-0.00062}^{+0.00015}$ & $50_{-21}^{+19}$ & $88.2_{-27.0}^{+25.8}$ & $14.44_{-0.45}^{+0.41}$ & $1395_{-436}^{+637}$ & $434_{-119}^{+15}$ & $1741_{-48}^{+69}$ & $1.02_{-0.05}^{+0.06}$ & $0.78_{-0.20}^{+0.06}$ & $\overline{1.33_{-0.29}^{+0.47}}$ \\
\hline 16803 & $234.994_{-0.026}^{+0.005}$ & $-2.098_{-0.011}^{+0.009}$ & $0.14845_{-0.00009}^{+0.00017}$ & $54_{-18}^{+13}$ & $94.9_{-29.6}^{+24.1}$ & $14.48_{-0.45}^{+0.40}$ & $1254_{-175}^{+333}$ & $445_{-76}^{+57}$ & $824_{-35}^{+297}$ & $0.90_{-0.07}^{+0.19}$ & $0.80_{-0.14}^{+0.18}$ & $0.59_{-0.06}^{+0.14}$ \\
\hline 19670 & $150.178_{-0.026}^{+0.028}$ & $50.784_{-0.007}^{+0.002}$ & $0.13442_{-0.00072}^{+0.000042}$ & $69_{-21}^{+24}$ & $87.0_{-24.8}^{+22.0}$ & $14.43_{-0.43}^{+0.40}$ & $1144_{-154}^{+606}$ & $494_{-91}^{+76}$ & $1651_{-158}^{+136}$ & $0.90_{-0.08}^{+0.09}$ & $0.91_{-0.11}^{+0.13}$ & $1.18_{-0.20}^{+0.54}$ \\
\hline 25124 & $243.538_{-0.015}^{+0.005}$ & $26.687_{-0.006}^{+0.002}$ & $0.18576_{-0.00009}^{+0.00107}$ & $30_{-14}^{+10}$ & $80.5_{-19.1}^{+24.5}$ & $14.40_{-0.43}^{+0.43}$ & $1380_{-521}^{+242}$ & $451_{-143}^{+13}$ & $1586_{-62}^{+19}$ & $0.95_{-0.09}^{+0.11}$ & $0.77_{-0.15}^{+0.20}$ & $1.24_{-0.35}^{+0.32}$ \\
\hline 32123 & $203.047_{-0.012}^{+0.020}$ & $45.425_{-0.039}^{+0.008}$ & $0.15958_{-0.00014}^{+0.00031}$ & $19_{-0}^{+17}$ & $32.8_{-0.7}^{+31.7}$ & $14.22_{-0.33}^{+0.59}$ & $837_{-0}^{+667}$ & $316_{-3}^{+129}$ & $1942_{-366}^{+1}$ & $0.97_{-0.12}^{+0.12}$ & $0.88_{-0.18}^{+0.10}$ & $2.00_{-0.92}^{+0.50}$ \\
\hline 36001 & $157.056_{-0.003}^{+0.019}$ & $21.339_{-0.031}^{+0.0398}$ & $0.18760_{-0.00012}^{+0.000001}$ & $6_{-1}^{+4}$ & $20.0_{-2.1}^{+12.3}$ & $13.79_{-0.36}^{+0.53}$ & $728_{-74}^{+334}$ & $194_{-24}^{+310}$ & $297_{-150}^{+1700}$ & $0.92_{-0.11}^{+0.12}$ & $0.57_{-0.08}^{+0.18}$ & $0.36_{-0.23}^{+0.46}$ \\
\hline 44564 & $208.170_{-0.012}^{+0.013}$ & $36.275_{-0.006}^{+0.005}$ & $0.14609_{-0.00009}^{+0.00006}$ & $114_{-38}^{+39}$ & $132_{-49.3}^{+58.0}$ & $14.62_{-0.50}^{+0.46}$ & $1336_{-200}^{+471}$ & $547_{-91}^{+84}$ & $1545_{-82}^{+126}$ & $0.87_{-0.06}^{+0.13}$ & $0.88_{-0.16}^{+0.19}$ & $0.99_{-0.32}^{+0.60}$ \\
\hline 44565 & $208.171_{-0.012}^{+0.012}$ & $36.277_{-0.007}^{+0.0004}$ & $0.14611_{-0.00006}^{+0.000006}$ & $115_{-35}^{+38}$ & $134_{-44.2}^{+57.0}$ & $14.62_{-0.47}^{+0.40}$ & $1336_{-183}^{+471}$ & $557_{-82}^{+71}$ & $1535_{-75}^{+115}$ & $0.88_{-0.07}^{+0.06}$ & $0.89_{-0.16}^{+0.16}$ & $1.00_{-0.32}^{+0.52}$ \\
\hline 44726 & $226.214_{-0.066}^{+0.001}$ & $29.833_{-0.064}^{+0.007}$ & $0.15233_{-0.00104}^{+0.00003}$ & $7_{-0}^{+15}$ & $11.0_{-0.4}^{+18.1}$ & $13.53_{-0.40}^{+0.70}$ & $188_{-10}^{+852}$ & $226_{-4}^{+2213}$ & $1331_{-6}^{+493}$ & $0.37_{-0.10}^{+0.05}$ & $0.94_{-0.14}^{+0.19}$ & $2.03_{-0.54}^{+0.83}$ \\
\hline 44739 & $229.822_{-0.019}^{+0.011}$ & $28.342_{-0.006}^{+0.0058}$ & $0.11883_{-0.00040}^{+0.00017}$ & $62_{-11}^{+44}$ & $65.0_{-6.7}^{+37.0}$ & $14.31_{-0.35}^{+0.40}$ & $1144_{-151}^{+658}$ & $348_{-63}^{+96}$ & $2394_{-416}^{+28}$ & $1.01_{-0.09}^{+0.06}$ & $0.71_{-0.10}^{+0.15}$ & $1.98_{-0.85}^{+0.64}$ \\
\hline 44858 & $230.084_{-0.032}^{+0.035}$ & $28.873_{-0.008}^{+0.000}$ & $0.12729_{-0.00024}^{+0.000082}$ & $80_{-28}^{+23}$ & $103_{-23.0}^{+22.0}$ & $14.52_{-0.42}^{+0.39}$ & $1313_{-419}^{+248}$ & $507_{-134}^{+74}$ & $2289_{-31}^{+172}$ & $0.87_{-0.05}^{+0.05}$ & $0.87_{-0.12}^{+0.05}$ & $1.61_{-0.43}^{+0.78}$ \\
\hline 50433 & $157.732_{-0003}^{+0.003}$ & $30.845_{-0.001}^{+0.0006}$ & $0.13630_{-0.00006}^{+0.00006}$ & $25_{-4}^{+7}$ & $39.7_{-30}^{+8.1}$ & $14.10_{-0.33}^{+0.38}$ & $600_{-38}^{+234}$ & $343_{-57}^{+27}$ & $1781_{-47}^{+11}$ & $0.62_{-0.08}^{+0.01}$ & $0.82_{-0.14}^{+0.11}$ & $1.73_{-0.42}^{+0.43}$ \\
\hline
\end{tabular}

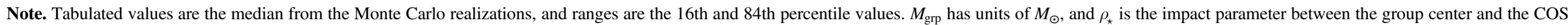
sight line. 


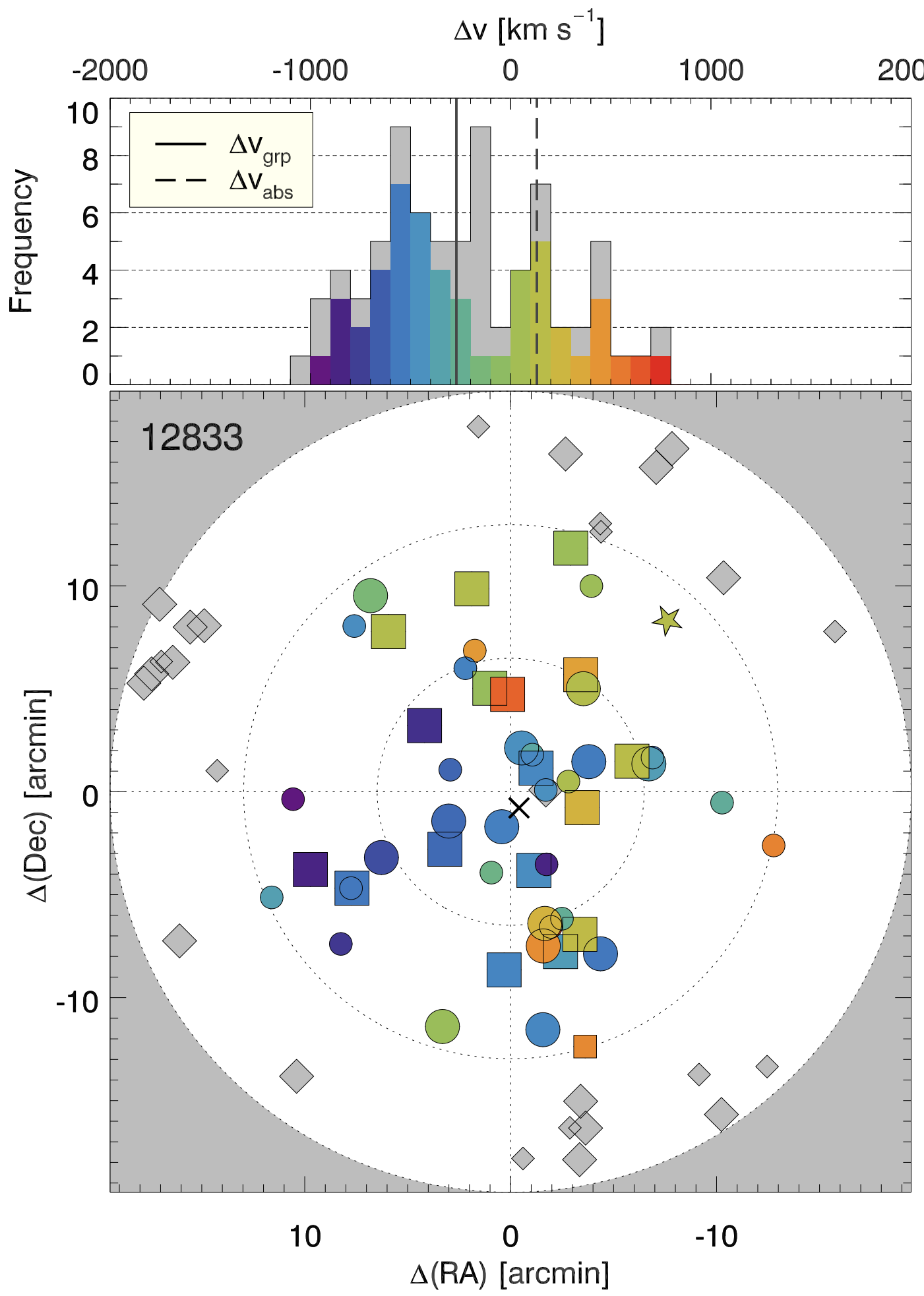

Figure 4. Top: Velocity histogram of galaxies near group 12833. Group members are color-coded from purple to red for the lowest and highest redshift group members, respectively. Field galaxies are gray. The solid vertical line shows the group velocity centroid, and dashed vertical lines show the positions of absorption in the HST/COS spectra. All velocities are calculated with respect to the initial estimate of the group redshift from the SDSS analysis in Table 2. Bottom: Distribution on the sky of galaxies near group 12833. Group members are colored as in the top panel. Gray diamonds represent field galaxies, squares represent SDSS galaxies, and circles represent new galaxy redshifts from our MOS campaign. Symbol size is indicative of galaxy luminosity, with the largest symbols indicating $L>L^{*}$, intermediate size symbols indicating $0.5 L^{*}<L<L^{*}$, and the smallest symbols indicating $L<0.5 L^{*}$. The position of the group centroid on the sky is marked with an " $\times$," and the position of the HST/COS sight line is marked with a star. Circles are shown at $1 \mathrm{Mpc}$ intervals, centered on the initial estimate of the group position from the SDSS analysis in Table 2.

(The complete figure set (12 images) is available.) 
Table 7

Group Members

\begin{tabular}{|c|c|c|c|c|c|c|c|c|}
\hline Name & $\begin{array}{l}\text { R.A. } \\
\text { (2) }\end{array}$ & $\begin{array}{l}\text { Decl. } \\
\text { (3) }\end{array}$ & (4) & $\begin{array}{c}L_{\text {gal }} \\
\left(L^{*}\right) \\
(5)\end{array}$ & Type & $\begin{array}{c}\Delta \rho \\
(\mathrm{kpc}) \\
(7)\end{array}$ & $\begin{array}{c}\Delta v \\
\left(\mathrm{~km} \mathrm{~s}^{-1}\right) \\
(8)\end{array}$ & $\begin{array}{c}f_{\text {mem }} \\
(\%) \\
(9)\end{array}$ \\
\hline \multicolumn{9}{|c|}{ Group 12833} \\
\hline rbs711_126_554 & 129.398389 & 44.325363 & 0.14572 & 0.575 & A & 323 & -316 & 99.6 \\
\hline rbs711_135_607 & 129.383140 & 44.296795 & 0.14519 & 0.998 & A & 265 & -455 & 99.5 \\
\hline 1237654652565651620 & 129.394131 & 44.314455 & 0.14505 & 1.66 & A & 261 & -492 & 99.5 \\
\hline rbs711_136_895 & 129.444666 & 44.229919 & 0.14614 & 0.519 & $\mathrm{C}$ & 627 & -207 & 99.3 \\
\hline rbs711_132_770 & 129.432936 & 44.267044 & 0.14492 & 1.20 & A & 272 & -525 & 99.3 \\
\hline
\end{tabular}

Note. Column 6 lists the galaxy's spectral type: "E" for emission-line galaxies, "A" for absorption-line galaxies, and "C" for composite galaxies that show both emission and absorption. $\Delta \rho$ and $\Delta v$ are calculated with respect to the original SDSS group centers in Table 2; $f_{\text {mem }}$ is the percentage of the Monte Carlo trials in which a galaxy was identified as a group member. Table 7, including the members of all 12 groups, is published in its entirety in the machine-readable format. A portion is shown here for guidance regarding its form and content.

(This table is available in its entirety in machine-readable form.)

Table 8

Adopted Group Properties

\begin{tabular}{|c|c|c|c|c|c|c|c|c|c|c|c|c|c|}
\hline $\begin{array}{l}\text { Group } \\
\text { (1) }\end{array}$ & $\begin{array}{l}\text { R.A. } \\
\left({ }^{\circ}\right) \\
(2)\end{array}$ & $\begin{array}{c}\text { Decl. } \\
\left(^{\circ}\right) \\
(3)\end{array}$ & $\begin{array}{l}z_{\text {grp }} \\
\text { (4) }\end{array}$ & $\begin{array}{r}N_{\text {grp }} \\
\text { (5) }\end{array}$ & $\begin{array}{c}\log M_{\text {grp }} \\
\text { (6) }\end{array}$ & $\begin{array}{c}R_{\mathrm{grp}} \\
(\mathrm{kpc}) \\
(7)\end{array}$ & $\begin{array}{c}\sigma_{\mathrm{grp}} \\
\left(\mathrm{km} \mathrm{s}^{-1}\right) \\
(8)\end{array}$ & $\begin{array}{c}R_{\mathrm{obs}} \\
(\mathrm{kpc}) \\
(9)\end{array}$ & $\begin{array}{c}\begin{array}{c}\sigma_{\mathrm{obs}} \\
\left(\mathrm{km} \mathrm{s}^{-1}\right)\end{array} \\
(10)\end{array}$ & $\begin{array}{c}\rho_{\star} / R_{\text {grp }} \\
(11)\end{array}$ & $\begin{array}{c}\Delta \rho \\
(\mathrm{kpc}) \\
(12)\end{array}$ & $\begin{array}{c}\Delta v \\
\left(\mathrm{~km} \mathrm{~s}^{-1}\right) \\
(13)\end{array}$ & $\begin{array}{c}f_{\text {spiral }} \\
(\%) \\
(14)\end{array}$ \\
\hline 12833 & 129.413 & 44.282 & 0.14589 & 50 & 14.45 & 1352 & 547 & 1396 & 444 & 1.32 & 137 & -271 & 43.9 \\
\hline 19670 & 150.178 & 50.785 & 0.13370 & 48 & 14.30 & 1205 & 487 & 991 & 404 & 1.36 & 128 & -193 & 50.0 \\
\hline 25124 & 243.538 & 26.687 & 0.18569 & 29 & 14.40 & 1301 & 526 & 1167 & 448 & 1.22 & 704 & -111 & 44.8 \\
\hline 32123 & 203.047 & 45.432 & 0.15958 & 19 & 14.01 & 965 & 390 & 837 & 313 & 2.01 & 268 & -34 & 50.0 \\
\hline 44565 & 208.170 & 36.280 & 0.14610 & 116 & 14.63 & 1553 & 628 & 1337 & 567 & 0.99 & 142 & -823 & 37.5 \\
\hline 44726 & 226.215 & 29.834 & 0.15236 & 7 & 13.53 & 667 & 270 & 178 & 223 & 1.98 & 527 & 74 & 40.0 \\
\hline 44739 & 229.828 & 28.336 & 0.11887 & 59 & 14.30 & 1205 & 487 & 1135 & 336 & 2.00 & 604 & 128 & 68.0 \\
\hline 44858 & 230.052 & 28.878 & 0.12707 & 99 & 14.59 & 1506 & 609 & 1546 & 569 & 1.49 & 364 & -88 & 50.0 \\
\hline 50433 & 157.732 & 30.845 & 0.13624 & 25 & 14.10 & 1034 & 418 & 600 & 338 & 1.73 & 187 & 67 & 31.3 \\
\hline
\end{tabular}

Note. $M_{\text {grp }}$ has units of $M_{\odot}$, and is calculated assuming $\Upsilon_{\text {grp }}=2.5 . \rho_{\star}$ is the impact parameter between the adopted group center and the COS sight line. $\Delta \rho$ and $\Delta v$ are offsets from the SDSS group centroids in Table 2. The final column lists an estimate of the group's spiral fraction derived from the spectral types of the adopted group members (see text for details).

range of virial radii of $R_{\mathrm{grp}}=650-1500 \mathrm{kpc}$ and velocity dispersions of $\sigma_{\text {grp }}=270-610 \mathrm{~km} \mathrm{~s}^{-1}$ (see Table 8). The range of impact parameters in this study is $0.36-2 R_{\text {grp }}$, with few sight lines $<R_{\text {grp }}$, due to the criterion that these sight lines could not intercept an SDSS galaxy halo at $<1.5 R_{\mathrm{vir}}$, naturally excluding sight lines in the dense, inner regions of groups.

There is little difference between the total halo masses computed by Paper III using just the SDSS spectroscopic survey galaxies and the total halo masses computed using the deeper MMTO redshift survey (see Table 8). The revised values are only marginally lower (statistically less by 0.05 dex or $12 \%$ ) than the SDSS values, much smaller than the factor of two to three combined errors on individual mass estimates (see Tables 2 and 6). Therefore, despite the modest number of group members in the SDSS analysis, the group halo masses derived by Paper III appear to be quite accurate and can be used as viable indicators of group mass for comparisons with other group properties, including those of the absorbers associated with groups at higher and lower estimated halo masses (see Sections 4.5, 5.1 and 5.2).

\subsection{Basic Group Properties}

In this section, the basic properties of these groups are explored in order to place the UV absorptions into their proper context. While the number of group galaxies $\left(N_{\mathrm{grp}}\right)$, the total group luminosity $\left(L_{\mathrm{grp}}\right)$, total group mass $\left(M_{\mathrm{grp}}\right)$, group virial radius $\left(R_{\mathrm{grp}}\right)$, and velocity dispersion $\left(\sigma_{\mathrm{grp}}\right)$ are all directly related by Equations (3)-(6), the observed velocity dispersion $\left(\sigma_{\text {obs }}\right)$ and the fraction of spiral or star-forming galaxies are independent of these derived group properties. The observed velocity dispersion differs from the calculated velocity dispersion $\left(\sigma_{\text {grp }}\right)$ because the group galaxies may not be in virial equilibrium.

In order to further explore the kinematic signature of virialization in these groups, Figure 5 shows the observed relationship between calculated group mass and the ratio between the observed and calculated velocity dispersion, $\sigma_{\text {obs }} / \sigma_{\text {grp }}$. At $M_{\text {grp }}>10^{14} M_{\odot}$, all but one of these groups have $\sigma_{\text {obs }} / \sigma_{\text {grp }} \geqslant 0.8$, suggesting that these massive groups are mostly virialized. The sample also includes a couple of groups 


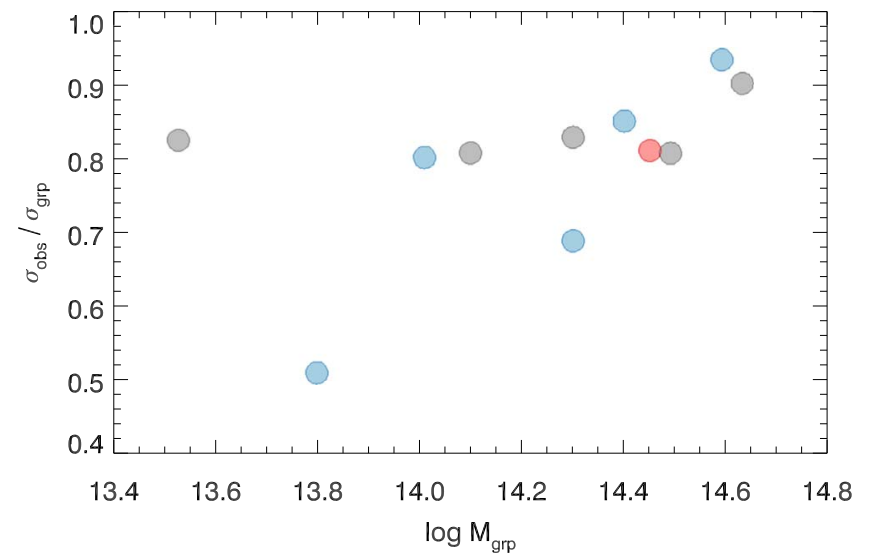

Figure 5. Calculated group mass $\left(M_{\mathrm{grp}}\right)$ vs. $\sigma_{\mathrm{obs}} / \sigma_{\mathrm{grp}}$, which is a measure of the virialization of these systems (e.g., $\sigma_{\mathrm{obs}} / \sigma_{\text {grp }}=1$ suggests a highly virialized system). Gray symbols are groups with only metal-free absorbers or nondetections in $\mathrm{H} \mathrm{I}$, while red symbols are groups with absorbers having only low ions present. O VI-bearing groups are blue. All but two groups have $\sigma_{\text {obs }} / \sigma_{\text {grp }} \gtrsim 0.8$; interestingly, the two less-virialized groups have rich associated UV absorption systems and high spiral fractions (discussed later).

(36001 and 44739) that are not close to being virialized by this same measure. These two groups are among the few in this sample with very rich absorption systems (e.g., group 44739 has 4 absorbers; one H I-only; one $\mathrm{HI}+\mathrm{Si}$ III; and two H I + O VI; see Section 5.2). Thus, this small sample appears to bridge the halo mass region where groups are becoming fully virialized in the current epoch.

Figures 6 and 7 show the spiral (i.e., star-forming) fraction of galaxies in these groups as a function of halo mass and of virialization, respectively. Because we do not possess good morphological information about these group galaxies, we have used the spectral classification of Keeney et al. (2018) as a proxy; in other words, emission-line and composite (emissionline plus absorption-line) galaxies are classified as starforming/spirals for this purpose. The spiral fraction is computed using all group members with $L>0.6 L^{*}$. The MMTO MOS group galaxy survey used for these classifications (Keeney et al. 2018) is $>90 \%$ complete to this limit out to $\rho_{\star} \leqslant 1 \mathrm{Mpc}$ for all groups except 25124, which is complete only to a limit slightly deeper than the SDSS $\left(\sim 3 L^{*}\right.$ out to $\left.\rho_{\star} \leqslant 1 \mathrm{Mpc}\right)$. If group 25124 , which is a massive $\left(10^{14.6} M_{\odot}\right)$, highly virialized $\left(\sigma_{\mathrm{obs}} / \sigma_{\mathrm{grp}}=0.8\right)$ group with $f_{\text {spiral }}=0.45$, were excluded from this discussion, it would not alter these plots noticeably.

It is clear from Figures 5 and 6 that the mass range of this survey spans a range of group properties from nearly fully virialized massive groups with large fractions of early-type galaxies (ETGs) to partially virialized groups with large spiral fractions. Figure 7 highlights that the spiral fraction and partial virialization go together, although this conclusion certainly requires a much larger group sample, particularly at lower halo mass and lesser virialization, to be robust. Nevertheless, in the most massive, highly virialized groups, the spiral fraction is $<50 \%$, so these groups are dominated by ETGs.

Among the nine groups with high virialization factors, the three groups with the lowest spiral fraction have no detected UV absorption lines. These groups are neither highly centrally concentrated nor dominated by a single ETG like the massive X-ray-detected groups of Mulchaey et al. (1996), but may have similar gas properties. Deep X-ray continuum observations are

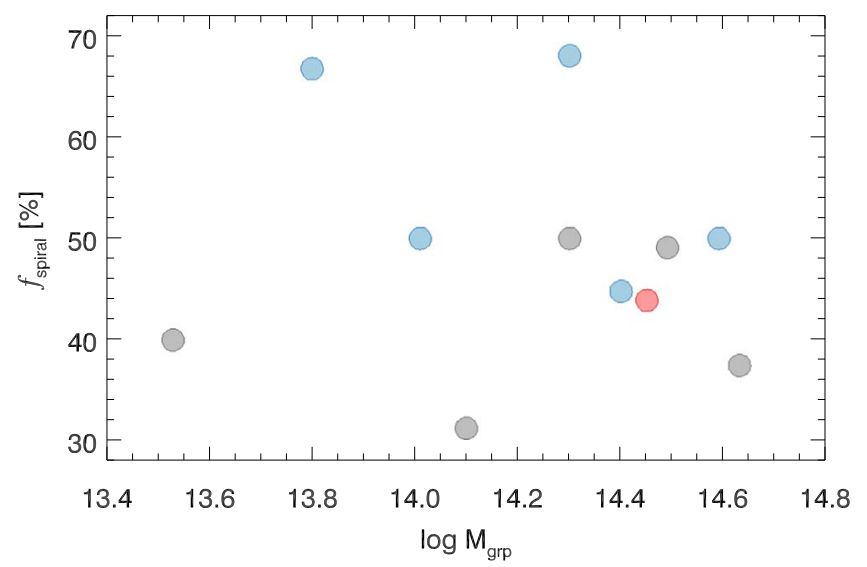

Figure 6. Calculated group mass $\left(M_{\mathrm{grp}}\right)$ vs. the fraction of $L>0.6 L^{*}$ group galaxies that are star-forming $\left(f_{\text {spiral }}\right)$. For the star-forming/spiral classification, we have used the spectroscopic proxy of emission lines; that is, all galaxy spectra with either emission lines only or emission lines plus absorption lines are classified as spirals for this purpose (Keeney et al. 2018). Gray symbols are groups with only metal-free absorbers or non-detections in $\mathrm{HI}$, while red symbols are groups with absorbers having only low ions present. O VI-bearing groups are blue.

appropriate to test whether a hot IGrM is present in the ETGdominated groups in this sample.

And while the least-massive groups in the sample are highly spiral-dominated and not always highly virialized, they are still an order of magnitude more massive than the small, spiraldominated groups, like the Local Group where most of the star formation in the current universe takes place. New UV spectroscopy of spiral-rich groups at $M_{\mathrm{grp}} \leqslant 10^{13.5} M_{\odot}$ is required to investigate typical star-forming sites in the local universe.

\section{Discussion of Results}

\subsection{Covering Fraction}

Despite the wide range of impact parameters targeted, H I absorption was detected toward 7 of the 12 groups probed through the full range of impact parameters (group 25124 is counted as a non-detection because its single absorber system is at least partly associated with an individual galaxy; see Table 5). Table 10 (see Appendix A) lists all absorbers within $\pm 2.5 \sigma_{0}$ (typically, $\pm 1000-1500 \mathrm{~km} \mathrm{~s}^{-1}$ ) of the group velocity centroid using the initial SDSS analysis (Table 2), but for the important choice of the absorber velocity range for group association, we adopt a range corresponding to $\pm 2.5 \sigma_{\text {grp }}$, based on the adopted group parameters listed in Table 8 .

While this choice is somewhat arbitrary, it is a good match to the criterion we used for including galaxies as group members; that is, based on mock catalogs produced from $N$-body simulations, $>99 \%$ of all satellite halos are found within $\pm 2 \sigma_{\text {grp }}$ of the primary halo's velocity (see Section 4.3). Increasing this range from $\pm 2 \sigma_{\text {grp }}$ to $\pm 2.5 \sigma_{\text {grp }}$ provides a small allowance for both the statistical sampling of the potential well using the modest number of galaxies available (i.e., uncertainties in mean velocity and velocity dispersion), and also for the peculiar velocity of gas which may have been expelled from, or is falling into, any individual galaxy in the group.

Enforcing these velocity bounds removes only one absorber system in Table 10 from group association: 36001/0.18429 at $-906 \mathrm{~km} \mathrm{~s}^{-1}$ (a velocity difference of $2.8 \sigma_{\text {grp }}$ ). While there are 


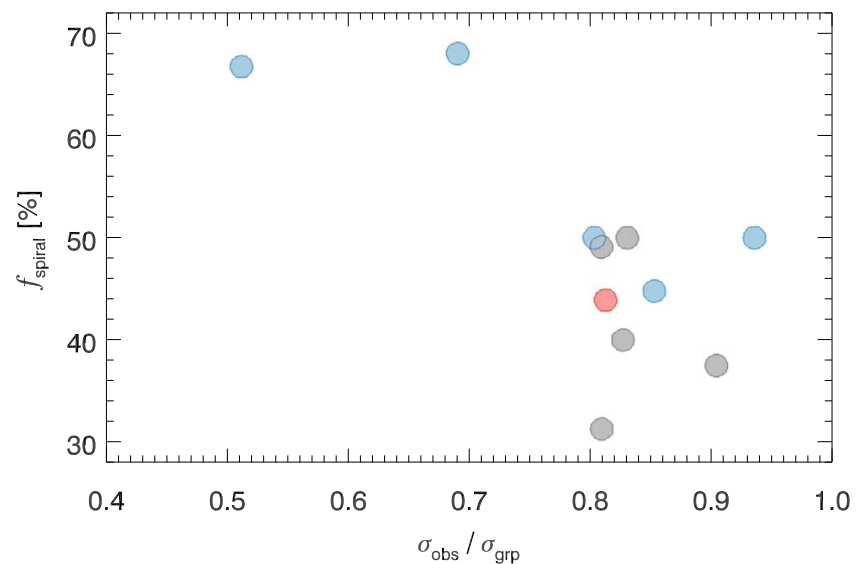

Figure 7. Virialization parameter $\left(\sigma_{\mathrm{obs}} / \sigma_{\mathrm{grp}}\right)$ vs. the fraction of $L>0.6 L^{*}$ group galaxies that are star-forming $\left(f_{\text {spiral }}\right)$. For the star-forming/spiral classification, we have used the spectroscopic proxy of emission lines; that is, all galaxy spectra with either emission lines only or emission lines plus absorption lines are classified as spirals for this purpose (Keeney et al. 2018). Gray symbols are groups with only metal-free absorbers or non-detections in $\mathrm{H} \mathrm{I}$, while red symbols are groups with absorbers having only low ions present. $\mathrm{O}$ VI-bearing groups are blue. In this plot, the strong correlation between ETGs and virialization is apparent, although more examples of spiral-rich, unvirialized groups are required to make this conclusion robust.

other absorbers that remain in the sample at larger velocity differences, this particular absorber is excluded because group 36001 has the smallest $\sigma_{\text {grp }}$ in the sample; however, there are two other absorber systems associated with group 36001, so it is retained as a group detection. Decreasing this limit to $2 \sigma_{\text {grp }}$ eliminates only an additional 2 systems of the 18 total. There are no viable individual galaxy associations associated with any of the systems with large $|\Delta v|$ (see Figure 1 and Table 5).

While there are 10 sight lines through 12 galaxy groups, as defined by our original set of observations, two modifications are required to correctly assess the covering fraction. First, our deeper MOS and subsequent analysis finds evidence that groups 44564 and 44565 in the CSO 1022 sight line are, in fact, one considerably more massive group; this leaves 11 groups probed. Second, the single absorber system in group 25124 was determined to be associated with a single bright galaxy, not the group as a whole; however, a BLA present within the Ly $\alpha$ absorption profile (see Section 3.1) is similar to other galaxy group absorptions investigated by Paper II. Due to this uncertainty, we eliminate group 25124 from the covering fraction discussion rather than count it as a non-detection, leaving 10 groups only.

The resulting Ly $\alpha$ covering fraction of the 10 groups of galaxies is shown in Table 9 as a function of normalized impact parameter, $\rho_{\star} / R_{\text {grp }}$. Despite the small number statistics, Table 9 shows that the covering fraction is substantial ( $\gtrsim 50 \%)$ out to at least $2 R_{\text {grp }}(\sim 2 \mathrm{Mpc}$, given the masses of the groups probed). This result is consistent with the rather constant distribution of covering fractions with radius ( $\sim 50 \%$ out to several virial radii, or $\sim 1 \mathrm{Mpc}$ ) seen for absorption-line galaxies in Keeney et al. (2018). It appears that the CGM of absorption-line galaxies joins seamlessly with group gas to which those galaxies typically belong.

Similarly, in Figure 8 the total H I column density within $2.5 \sigma_{\text {grp }}$ of $z_{\text {grp }}$ along these group sight lines is shown as a function of sight line impact parameter from the group centroid on the sky. The obvious conclusion from this plot is that cool

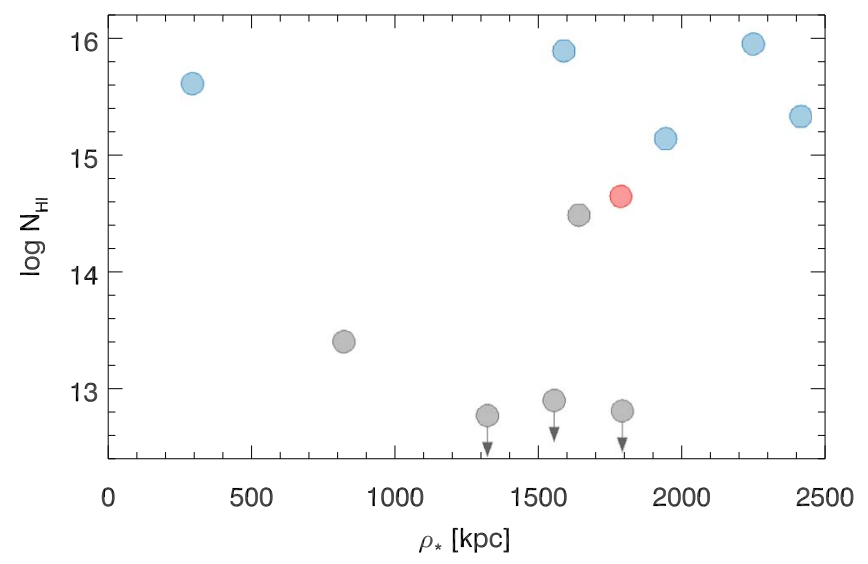

Figure 8. Total $N_{\mathrm{H}}$ in all absorption components associated with the group as a function of the physical impact parameter $\rho_{\star}$. Gray symbols are groups with only metal-free absorbers or non-detections in $\mathrm{HI}$, while red symbols are groups with absorbers having only low ions present. O VI-bearing groups are blue. The H I column densities do not show any obvious trend with the groupcentric impact parameter out to $2 \mathrm{Mpc}$, or $\sim 2 R_{\text {grp }}$.

Table 9

Covering Fraction of Group Absorption

\begin{tabular}{ccl}
\hline \hline$\rho_{\star} / R_{\text {grp }}$ & Cov. Frac. & Notes \\
\hline $0.0-0.5$ & $1 / 1$ & All have O VI \\
$0.5-1.0$ & $1 / 2$ & Metal-free \\
$1.0-1.5$ & $2 / 2$ & One metal-free, one low ions only \\
$1.5-2.0$ & $3 / 5$ & All have O VI \\
\hline
\end{tabular}

and/or warm gas remains abundant out to at least $2 \mathrm{Mpc}$ (or $\sim 2 R_{\text {grp }}$ ) for these groups. While the high but $<100 \%$, covering fraction found here may be due to a patchy distribution of UVabsorbing gas in all these groups, it is also possible that the presence or absence of this gas can be due to other factors.

In Paper II, an argument was presented that the area covered by warm group gas is large, $\sim 1 \mathrm{Mpc}$ in radius per group if unity covering factor is assumed. This estimate is based on the number density per unit redshift $(d \mathcal{N} / d z=4)$ of warm absorbers $\left(T \geqslant 10^{5} \mathrm{~K}\right)$ seen at low- $z$ and the space density of groups of galaxies $\left(\approx 3 \times 10^{-4} \mathrm{Mpc}^{-3}\right.$; Paper III). The $\gtrsim 50 \%$ covering fraction out to $\rho \geqslant 2 \mathrm{Mpc}$ found by this study supports the previous conclusion that a large gaseous medium exists in galaxy groups.

However, the huge area covered by $\mathrm{HI}$ absorption does not require a huge mass because the filling factor of this gas is unknown and could be small; for example, if the cool gas is in small clouds like in the CGM and the warm gas is in filamentary structures scattered throughout the volume of the group, the filling factor of cool plus warm group gas could be similar to the filling factor (3\%-5\%) of cool gas in the CGM, or even smaller. In this case, the UV-detected group gas can have a total gas mass per galaxy of only $2 \%-4 \%$ of the full baryon budget. This makes it unlikely that the UV-detected group gas solves the baryon problem. But, when viewed as transition gas, the UV-absorbing gas may be cospatial with even hotter gas that constitutes a volume-filling IGrM. Further, in this sample there is no strong evidence for warm or warm-hot gas found associated with these groups (see Section 3.2).

Figure 9 shows the total H I column density of all absorption components in these groups as a function of group mass. Despite 


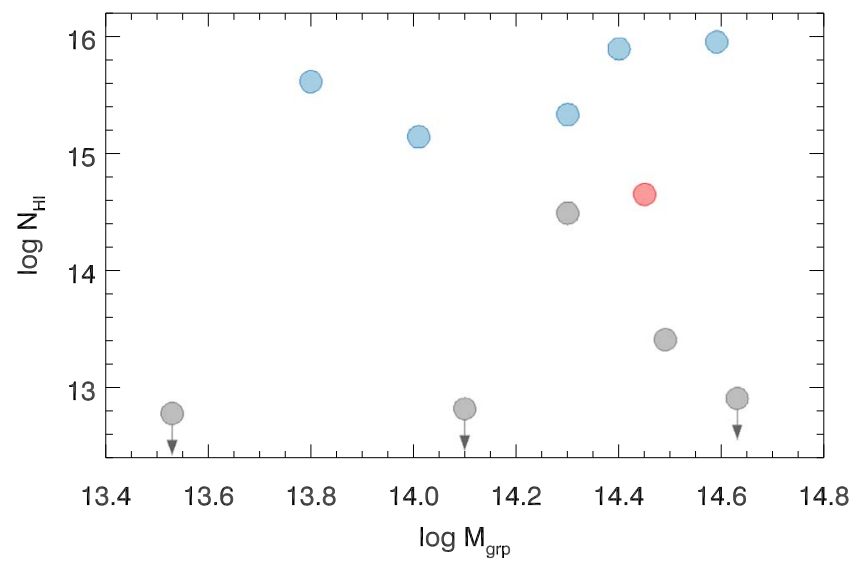

Figure 9. Total $N_{\mathrm{HI}}$ in all absorption components associated with the group vs. the calculated group mass $\left(M_{\mathrm{grp}}\right)$. Gray symbols are groups with only metalfree absorbers or non-detections in $\mathrm{HI}$, while red symbols are groups with absorbers having only low ions present. O VI-bearing groups are blue. Despite including both virialized and unvirialized groups, there is no obvious trend between the presence of absorbers and total group halo mass in this sample. Neither the H I detections/non-detections nor the H I column densities show any obvious trend with the estimated mass of the group.

their lower mass, the two relatively unvirialized groups, 36001 $\left(\log M_{\text {grp }}=13.80\right)$ and $44739\left(\log M_{\text {grp }}=14.30\right)$, both have H I-rich absorption systems. There is no obvious trend between group mass and either detections/non-detections or the total $N_{\mathrm{H} \mathrm{I}}$ in this mass range.

However, it is notable that the three groups that lack H I absorption (Table 10 in Appendix A) are the three most ETGrich groups (Table 8), and among the most highly virialized (Figure 7). While a larger sample is certainly needed, this result suggests that groups which are spiral-rich and not completely virialized will be detected strongly and multiply in $\mathrm{HI}$ and metals. This result predicts that less-virialized groups at lower halo mass will be found to have large reservoirs of cool plus warm UV-detectable gas, which is not obviously associated with a specific group galaxy.

Different from the results found in Paper II, there are very few (two probable and four possible) warm absorbers found in these groups. Despite the results of Paper II, this suggests that the warm gas in groups is rather patchy and with potentially low covering fraction (i.e., $\lesssim 10 \%$ ).

\subsection{Absorber Kinematics}

Limited by our available measurement tools, the kinematic information we have for these absorbers consists of (1) a twodimensional location on the sky with respect to the sky centroid of the group, and (2) the radial component of the velocity relative to the group centroid velocity. In Figure 10, these basic quantities are displayed in a plot of impact parameter, $\rho_{\star}$, versus the onedimensional velocity difference, $\Delta v=c\left(z_{\mathrm{abs}}-z_{\mathrm{grp}}\right) /\left(1+z_{\mathrm{grp}}\right)$. There is no strong concentration around $\Delta v=0$, which would be expected if the detected gas is a massive IGrM as seen in X-ray bremsstralung emission for more massive systems (Mulchaey 2000). The relative dearth of absorbers at $\rho_{\star} \leqslant 1.5 \mathrm{Mpc}$ is due to our imposed criterion that no sight line pass within $1.5 R_{\mathrm{vir}}$ of an SDSS group galaxy, thus avoiding the dense central regions of groups.

There is no obvious kinematic difference between the metalbearing absorbers (red symbols for only low ions present and blue symbols for O VI present) and the metal-free absorbers

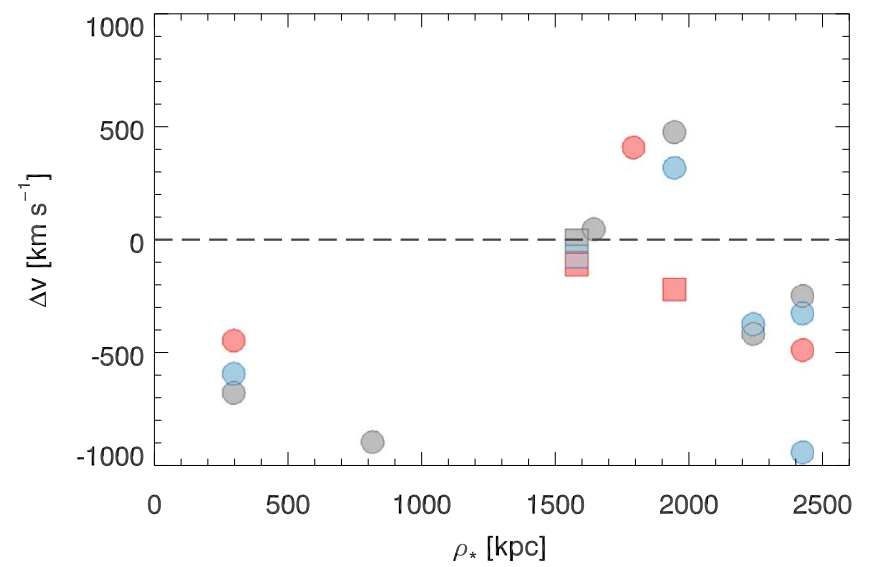

Figure 10. The physical impact parameter in $\mathrm{kpc}$ is plotted against the (absorber-group) velocity difference in $\mathrm{km} \mathrm{s}^{-1}$. Colors are as in Figure 9, and squares are absorbers, which could be associated with individual galaxies.

(gray symbols); nor is there any difference between the locations of the high and low ions. The square symbols show the location of the absorbers possibly associated with individual bright galaxies in groups 25124 and 32123. If any or all of those absorbers are associated with the entire group rather than individual galaxies, the basic results of this plot are not altered.

More revealing is Figure 11, which shows these same observables normalized by the theoretical quantities of group virial radius (i.e., $\rho_{\star} / R_{\text {grp }}$ ) on the horizontal axis and escape velocity (i.e., $|\Delta v| / v_{\text {esc }}$ ) on the vertical axis, where the escape velocity is calculated at a radius equal to the observed impact parameter. What is striking in this plot is that only one of the absorbers has sufficient radial velocity to escape the group gravitational potential well.

This result is similar to the results found by the COS-Halos study (Tumlinson et al. 2011) of the inner half of the CGM for which absorbers do not possess sufficient radial velocity to escape their home galaxy's gravitational potential. While Stocke et al. (2013) and Keeney et al. (2017) found a similar result to COS-Halos in the inner CGM, when the outer CGM $\left(\rho>0.5 R_{\text {vir }}\right)$ is probed, the results are quite different. At $\rho>0.5 R_{\text {vir }}$, the one-dimensional velocity difference between absorbers and their associated galaxies cluster around the value for the escape velocity (see Figure 8 in Stocke et al. 2013 and Figure 13 in Keeney et al. 2017). For individual galaxies, this suggests that many of these CGM absorbers will escape if their direction of motion is out-going, although some few still are unlikely to escape (see, e.g., Tumlinson et al. 1999, 2011; Keeney et al. 2005; Stocke et al. 2010, 2013). Presumably the absorbers discovered in this small survey are examples of gas that has escaped its home galaxy CGM and is now bound to its home galaxy group.

This result does not appear to be caused by the limit placed on absorber velocity, by which absorbers are excluded for association if $|\Delta v|>2.5 \sigma_{\text {grp }}$ from the group mean velocity. The one absorber excluded by this velocity cut (36001/ 0.18429 ) has a radial velocity relative to this group of only $0.7 v_{\text {esc }}$. On the other hand, the absorber whose relative velocity exceeds the escape speed $(44739 / 0.11537$, a mixed H I, O VI, and Si III absorber) has a relative velocity of $<2 \sigma_{\text {grp }}$ from the mean group velocity and so would have been included even if the limit was lowered. 


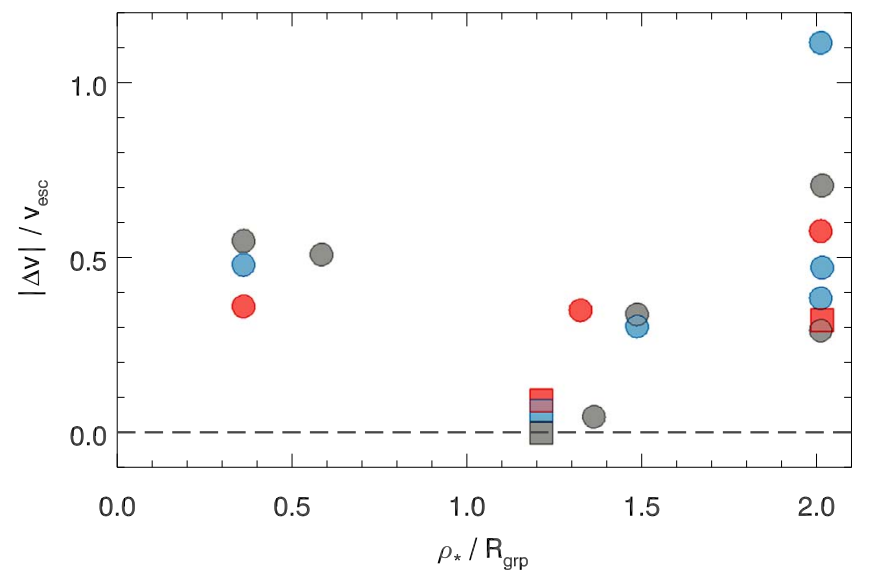

Figure 11. The symbols are similar to their depictions in Figure 10, but in this case the impact parameter $\left(\rho_{\star}\right)$ is displayed in units of the group virial radius $\left(R_{\mathrm{grp}}\right)$, while the velocity difference is in units of the escape velocity $\left(v_{\mathrm{esc}}\right)$ at the radius of the impact parameter.

To ensure that any fast-moving absorber that could have escaped from these groups has not been excluded from consideration by the assumed cutoff in velocity that was imposed, a search for such absorbers was conducted around all the sight lines in this survey. Increasing the relative velocity bounds to include all absorbers at $|\Delta v| \leqslant 5 \sigma_{\text {grp }}$ (roughly $\pm 2500 \mathrm{~km} \mathrm{~s}^{-1}$ ) from the group redshift, an additional dozen absorbers were found; however, all but three of these are metalfree and not relevant to these concerns. Of the remaining three, two show absorption in $\mathrm{HI}$ and $\mathrm{O}$ VI, and are located only $0.83 R_{\text {vir }}$ from the same $0.5 L^{*}$ galaxy, an individual galaxy association by our earlier discussion. The third absorber is $2.3 R_{\text {vir }}$ from a $2 L^{*}$ galaxy near group 19670 , so this absorber could be associated with a lower luminosity galaxy not observed in our program, or it could be associated with another group of galaxies to which this nearest galaxy is a member, or it has been ejected from group 19670 at $\sim 1800 \mathrm{~km} \mathrm{~s}^{-1}$, or $1.5 v_{\mathrm{esc}}$. Even if the latter is correct, only 2 of 13 metal-bearing absorbers in these groups could have relative radial velocities exceeding the escape velocity for the groups they probe; thus any group mass lost by this process to the IGM is small.

Reinforcing the results shown in Figures 10 and 11 is Figure 12, which shows that there is little trend between the normalized absorber-group velocity difference and the group mass. Except for the one absorber mentioned previously, which exceeds the escape speed in radial velocity difference, there is little evidence that these absorption systems will escape from their parent groups.

For galaxy groups, Figure 11 shows that most absorbers may not escape from the group potential unless their direction of motion is mostly tangential on the sky or inward. This result can have significant implications for galaxy evolution (e.g., these groups are close to being "closed boxes" for galactic evolution in the current epoch). While some gas can be accreted onto the group from the intergalactic medium, any metals currently produced by group galaxies may not leave the group. Any baryons and metals that left the region of this group must have done so at much earlier cosmic times.

The BAHAMAS cosmological hydrodynamic simulations (McCarthy et al. 2017), which have been tuned to observational $\mathrm{X}$-ray and Sunyaev-Zeldovich Effect constraints for groups and

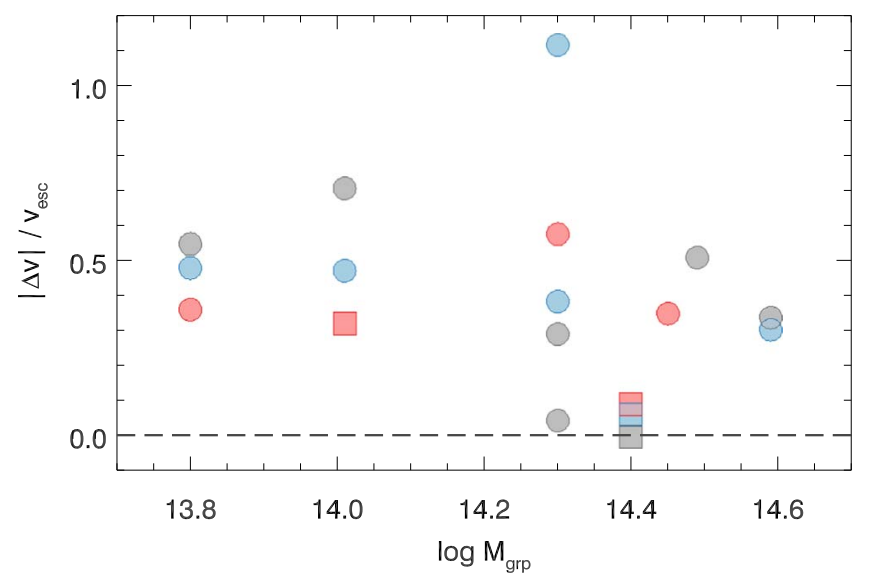

Figure 12. Plot of the calculated group mass $\left(M_{\text {grp }}\right)$ vs. the absorber-group radial velocity difference, where the velocity difference is in units of the escape velocity $\left(v_{\mathrm{esc}}\right)$ at the radius of the impact parameter.

clusters, predict that about one-third of the baryons associated with a $10^{13.8} M_{\odot}$ halo mass are ejected beyond the virial radius, while a $10^{14.6} M_{\odot}$ halo should retain almost all of its bayons and be considered essentially baryonically closed. Almost all of the groups here are at estimated halo masses in excess of $10^{14} M_{\odot}$, in the regime where the BAHAMAS simulations suggest that most of the baryons should be retained by the group, suggesting that a hot, massive IGrM is present in these groups.

\subsection{Gas versus Group Properties}

In our initial study of gas in galaxy groups (Paper II), we used previously detected H I plus O VI absorbers from Paper I as markers to search for groups at the absorber redshift. Groups of varying richness (total group luminosities ranging from a few $L^{*}$ to nearly $100 L^{*}$ ) were found near these absorbers. In that study, weak correlations were seen between gas and group properties, which supported the identification of those absorbers with the groups distinct from gas associated with individual galaxies in the groups.

Herein, the selection criteria excluded sight lines near ( $\rho \leqslant 1.5 R_{\text {vir }}$ ) bright galaxies in these groups, strongly supporting a group association for these absorbers. Still, many (nine) absorbers were found within these groups with low ions like Si III typical of cool, photoionized CGM absorbers. Only two of these apparently photoionized absorbers are at $\rho<1.5 R_{\text {vir }}$ from the nearest galaxy; seven others are at larger distances, including three at $\rho>3 R_{\text {vir }}$ away.

While it is possible that there are sub- $L^{*}$ galaxies closer to some of these absorbers that were not observed by our galaxy survey (Keeney et al. 2018), more likely these metal-bearing clouds have escaped their home galaxy's potential well and are now bound within the confines of their home galaxy group. The cool clouds in these groups that are well away from galaxies could be CGM clouds that have escaped their home galaxy as predicted by Stocke et al. (2013) and Keeney et al. (2017), based on CGM cloud kinematics.

Two plots from Paper II suggested weak correlations between absorber temperature and total group luminosity (Figure 13) and between absorber temperature and group velocity dispersion (Figure 14). These are reproduced here with the addition of a few points from this study selected in the same way; that is, O VI absorbers in groups with either warm or cool 


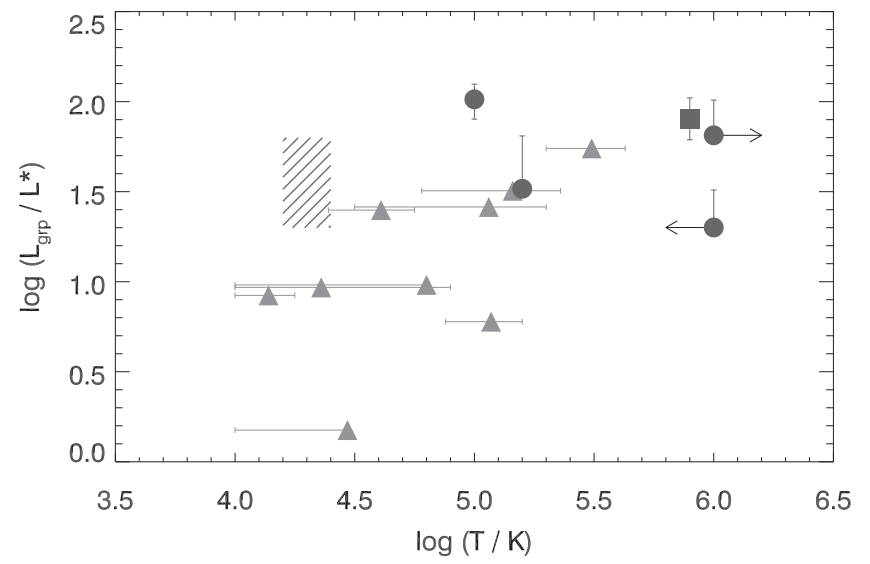

Figure 13. Group absorber temperatures vs. total group luminosity, reproduced from Figure 10 of Paper II. The gray points are from Paper II, while the black points are warm absorbers from this study. The current sample temperatures are all quite uncertain and should be viewed as estimates. The hashed area is the approximate location for four photoionized group absorbers from this study. With the addition of the new data, there is no obvious correlation found between these parameters.

absorbers depending on $\mathrm{HI}, \mathrm{O}$ VI, and $\mathrm{Si}$ III properties (see Section 3.2). For these figures, we have used the possible warm absorber temperatures derived in Section 3.2 to provide the maximum possibility for showing correlations in these plots. These figures show that any correlation between these quantities is very weak or nonexistent. In these two figures, five points from the current study are added at $T>10^{5} \mathrm{~K}$ (group 32123 has two possible warm absorbers; the one with a measured value is plotted, not the upper limit). The crosshatched region shows the range of cool absorber properties where $T \approx 20,000 \mathrm{~K}$ is assumed. If no warm absorbers are present in this sample, as appears likely based on the results presented in Section 3.2, the possibility for correlations in these two plots is diminished further.

While the groups studied here are comparable to only the most luminous and massive groups found by Paper II, these groups possess gas at the full range of temperatures from $20,000 \mathrm{~K}$ to $>10^{6} \mathrm{~K}$, diminishing the already marginal correlations found by the earlier work. Further, the absence of correlations in Figures 13 and 14 show that the gas temperature is not directly related to the mass of the group (i.e., its potential well depth). Nor are the absorbers detected at velocities close to the group centroid velocities. These are other indicators that the UV-detected gas is not volume filling but is, instead, that gas most easily detectable through UV absorption.

Since the possibly warm clouds have temperatures too hot to be photoionized by the UV background, either these absorbers are heated by interaction with a hotter medium or are condensing out of it. In either case, a hot IGrM is suggested to be present that could be connected more intimately to the gravitational potential well depth. In richer systems like clusters and elliptical-dominated groups, this hotter gas is detected in X-ray bremsstrahlung emission.

Based on extrapolations from the richer systems shown in Figure 14, the groups studied here are expected to have an IGrM temperature of $\log (T / \mathrm{K})=6-7$, much hotter than the warm clouds we detect as BLAs and broad O VI absorbers. The solid line extrapolation assumes energy equipartition between gas and galaxies (i.e., $\beta_{\text {spec }}=1$; Osmond \& Ponman 2004),

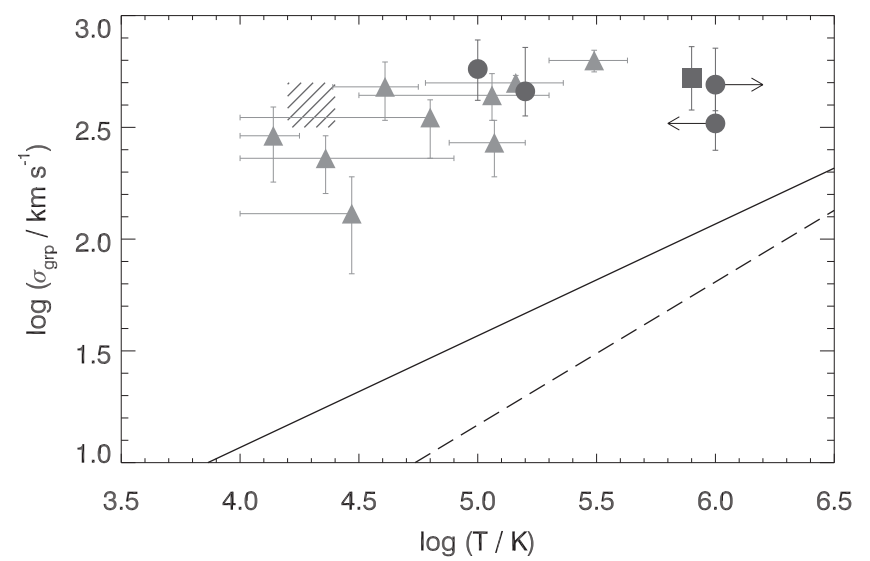

Figure 14. Group absorber temperatures vs. group velocity dispersion, reproduced from Figure 11 of Paper II. The gray points are from Paper II, while the black points are warm absorbers from this study. The current sample temperatures are all quite uncertain and should be viewed as estimates. The hashed area is the approximate location for four photoionized group absorbers from this study. With the addition of the new data, there is no obvious correlation found between these parameters. The solid line is the extrapolation of $\beta_{\text {spec }}=1$ (energy equipartition between galaxies and gas) from the clusters and groups data found in Osmond and Ponman (2004); see their Figure 16. The dashed line is an extrapolation of the best-fit power law for clusters and groups from Wu et al. (1999). Notice that the points and limits for the warm absorbers in groups are far above either of these extrapolations from richer galaxy systems, illustrating that this gas is too cool by one order of magnitude or more from the expected volume-filling intra-group gas conditions.

while the dashed line is the best-fit power-law extrapolation based on the Wu et al. (1999) compilation of clusters and groups. Our data points in Figure 14 are clearly too cool to fall near these extrapolations, nor do the current data possess the slope appropriate for an IGrM based on these extrapolations.

\section{Summary and Conclusions}

This study uses UV absorption lines detected in COS/ G130M spectra of 10 AGN targets that probe 12 galaxy groups selected from a homogeneous catalog culled from the SDSS spectroscopic survey in the range $z_{0}=0.10-0.19$, which facilitates the detection of both the O VI doublet and Ly $\alpha$ in the single G130M exposures allocated. These sight lines occur at group-centered impact parameters ranging from 0.3 to 2 group virial radii with absorber-group radial velocity differences as large as $\pm 1000 \mathrm{~km} \mathrm{~s}^{-1}$.

In order to ensure that these spectra probe gas associated with the foreground groups distinct from individual galaxies within the groups, an important sample selection criterion is that no sight line passes within 1.5 virial radii of a bright SDSS group galaxy. Earlier work (Keeney et al. 2017) showed that an association between an absorber and a single galaxy is plausible only if the impact parameter $\rho \leqslant 1.4 R_{\mathrm{vir}}$. But this selection criterion also has the effect of minimizing the number of sight lines close to group centers where the galaxy density is highest, minimizing sample statistics close to group centers.

Excepting for SDSS J1540-0205 (where a LLS at $z=0.33$ obscures the $\mathrm{O}$ VI region in group 16803), $\mathrm{S} / \mathrm{N}=15-30$ was achieved for these targets in the wavelength regions of $\mathrm{Ly} \alpha$ and the O VI doublet at the group redshift. Absorption associated with these groups was detected for 7 of the 12 groups probed, including group 16803, where only Ly $\alpha$ was detected. Metal lines, usually the O VI doublet and/or Si III $1206 \AA$, are seen in 
12 of the 18 absorber components detected. See Figure 1 for the COS spectra; all line parameters are listed in Table 10.

These HST/COS observations were supported by a deep and wide galaxy redshift survey around each of these sight lines (see Keeney et al. 2018 for details of these observations and subsequent spectral analysis). While the SDSS spectroscopic survey provides complete galaxy redshift information down to $L \gtrsim L^{*}$, the MOS obtained by Keeney et al. (2018) extends to $L>0.2 L^{*}$ at a completeness level over a $20^{\prime}$-radius field-ofview of $\gtrsim 60 \%$. Completeness levels for this galaxy redshift survey are substantially better ( $\gtrsim 75 \%)$, both for faint galaxies $(g \leqslant 20)$ close $(\lesssim 700 \mathrm{kpc})$ to the sight line and for luminous group galaxies out to large radii $(<2.5 \mathrm{Mpc})$.

These MOS observations were designed to obtain $\geqslant 20$ group members so that each group could be correctly characterized from the galaxy data. This observing goal was accomplished in all but three cases (groups 36001, 49980, and 44726), which have few member galaxies in total, not because they were poorly observed. The galaxy positions and redshifts provide ample data to characterize these groups using a unique Monte Carlo approach that is described in detail in Section 4. All MOS galaxy redshifts used in this study can be found at MAST via doi:10.17909/T9XH52. ${ }^{9}$

The resulting group parameters from the Monte Carlo process are listed in Tables 6 and 8, and are generally very similar to the parameters found by the algorithm of Paper III using the SDSS data alone Table 2. These groups have varying richness with total luminosities $L=10-130 L^{*}$, virial radii of $R_{\text {grp }}=680-1540 \mathrm{Mpc}$, velocity dispersions of $\sigma_{\text {grp }}=270$ $630 \mathrm{~km} \mathrm{~s}^{-1}$, and estimated halo masses of $\log \left(M_{\text {grp }} / M_{\odot}\right)=$ 13.5-14.5. At these masses, the groups in this sample have a mixture of early- and late-type galaxies and are more massive than most nearby groups in the Local Supercluster, including the Local Group. Additionally, this sample spans the range from almost fully virialized groups dominated by early-type, passive galaxies to partially virialized, spiral-rich groups, as determined by new analysis tools developed for this study and described in Sections 4.3-4.5. These groups are similar to the groups in which Paper II found warm absorbers.

Based on the information derived from these two data sets, the basic results from this study include the following:

1. The covering fraction of $\operatorname{Ly} \alpha$ absorption due to these groups is $\gtrsim 50 \%$ at impact parameters $\rho=0.4-2 R_{\text {grp }}$ (see Table 9 in Section 5.1, showing the small number statistics for this result); $R_{\mathrm{grp}} \approx 1 \mathrm{Mpc}$ for the masses of these groups. Additionally, there is no obvious trend between the observed $N_{\mathrm{H} \text { I }}$ and impact parameter out to $\approx 2 \mathrm{Mpc}$. This is quite different from the rapidly declining CGM $N_{\mathrm{H} \text { I }}$ around individual star-forming galaxies. While large covering fractions over such a huge area around these groups is consistent with the number density of warm absorbers $(d \mathcal{N} / d z=4$ per unit redshift; Paper II), high covering fractions do not lead necessarily to large volume-filling factors and very large masses of cool and/ or warm gas (discussed later). However, unlike the results found by Paper II, in this sample there are few demonstrably warm absorbers, suggesting that warm gas specifically could have a rather small covering fraction in massive galaxy groups. Given the unstable condition of warm, collisionally ionized gas, it is likely

9 https://archive.stsci.edu/prepds/igm-gal/ that this gas exists in filaments that fill only a few percent of the group's volume and account for only a few percent of the total baryon budget of the group.

2. While the H I covering fraction of these groups is high over a very large surface area, it is possible that a few massive groups always possess detectable warm and/or cool $\mathrm{H}$ I absorption and others do not. In this sample, the three HI non-detections are all quite massive, highly virialized groups with spiral galaxy fractions of $\sim 40 \%$ (see discussion in Section 5.1). This result leads to the prediction that lower-mass (approximately one order of magnitude less massive that those studied here), spiralrich groups will have $\mathrm{H}$ I Ly $\alpha$ detections at close to $100 \%$ covering fraction out to a couple of virial radii in impact parameter. Because these lower halo mass groups, which are similar to the Local Group in mass and kinematics, are the sites where most of the current-epoch star formation occurs, probing these groups for UV absorption is vital to understand the full context of star formation in the local universe.

3. Different from the circumstance for single-galaxy CGMs, the absorbers associated with groups do not possess sufficient relative radial velocities to escape from these groups (i.e., $|\Delta v| / v_{\text {esc }} \leqslant 0.6$, except for one absorber with $|\Delta v|=1.1 v_{\text {esc }}$; see Section 5.2). Because seven H I and low-metal-ion (usually Si III) absorbers are found in these groups well away from individual galaxies, cool CGM clouds do escape from their home galaxy (as suggested by CGM cloud radial velocities close to $v_{\text {esc }}$; see Stocke et al. 2013; Keeney et al. 2017) but do not escape from their home galaxy group. This has the important consequence for massive galaxy groups in the mass range studied here that little or no gas escapes from galaxy groups into the IGM in the current epoch. If baryons and metals have left the region of this group, that process must have occurred at earlier cosmic times.

4. There are several observational results from this study that strongly suggest that the cool and/or warm gas detected in groups is neither volume filling nor massive. These observational results include (1) the cool or warm gas detected is not close to either the sky centroid of these groups nor to their radial velocity centroid (see Figure 10); (2) the temperature of this gas does not correlate with two different measurements of group halo mass, its total luminosity (see Figure 13), and the velocity dispersion of its galaxies (see Figure 14); and (3) the warm gas temperatures, both those possible detections found herein and those found in Stocke et al. (2014), are too cool by on order of magnitude to be a volume-filling IGrM based on extrapolations from richer galaxy systems (see Figure 14).

While plentiful warm and cool gas has been found associated with rich groups of galaxies, a massive IGrM that completes the baryon inventory in star-forming and passive galaxies has yet to be discovered. Given its predicted temperature range of $10^{6}-10^{7} \mathrm{~K}$, new soft X-ray instrumentation and telescopes will be required to discover it. Based on assuming pressure balance with individual CGM clouds at $P / k \approx 10 \mathrm{~cm}^{-3} \mathrm{~K}$, an IGrM at these temperatures has physical densities of $n=10^{-5}$ to $10^{-6} \mathrm{~cm}^{-3}$, making its detection challenging (although see Nicastro et al. 2018 and M. Donahue et al. 2019, in preparation, for tentative detections). 
With little evidence for gas escaping from these groups, systems with total halo masses of $10^{13.5}-10^{14.5} M_{\odot}$ should be considered "closed boxes" in the current epoch for galactic evolution and the baryon census. Since the currently detected baryons are insufficient to bring these groups up to the cosmic ratio of baryons to dark matter, a massive IGrM is likely present. Indeed, the recent BAHAMAS numerical simulations predict that most of the groups studied here, specifically the most massive ones, have retained most of their baryons. If this is the case, a massive IGrM should be present in these groups to close the baryon accounting. Efforts should be made to extend these UV results downward in halo mass to systems the size of the Local Group and determine if these smaller groups show similar trends (e.g., the presence of both cool and warm gas and few absorbers escaping). These lower-mass groups are important because they are the sites where most of the star formation in the current universe is taking place. Since different galaxy groups can have differing evolutionary and thus metallicity histories (e.g., only some have had major mergers early on), the galaxy metallicity pattern in groups can vary. This may be observable as differences in the mass-metallicity relationship in different groups, contributing to the width observable in that relationship when galaxies from many groups are included (factor of $\sim 4$ [95\% contour] in metallicity at fixed mass; Tremonti et al. 2004).

This study also clarifies that theoretically modeling the CGM of star-forming and passive galaxies must account for the IGrM of the groups in which the absorbers and galaxies are found. Specifically, this means that the halo masses investigated in numerical simulations must concentrate on group-size halos $\left(10^{12.5}-10^{14.5} M_{\odot}\right)$ both to fully account for the all the baryons in these systems and to predict CGM and IGrM properties successfully.

This research was supported by NASA/HST grants 14277 (PI: JTS) and 14308 (PI: BDO). Special thanks to Alex Parker for stimulating discussions. Access to the MMTO through the University of Arizona is gratefully acknowledged.

\section{Appendix A \\ Notes on Absorption toward Individual Sight Lines}

Table 10 lists the species, redshift, velocity offset with respect to the SDSS group redshift, significance level, equivalent width, Doppler $b$-value, and column density of all absorbers detected within $\pm 1000 \mathrm{~km} \mathrm{~s}^{-1}$ of the SDSS group redshifts. Figure 1 shows the portions of the COS/G130M spectra over approximately the same range. The absorption toward each sight line is discussed as follows.

\section{A.1. Group 12833; $\mathrm{z}_{0}=0.14693, \operatorname{RBS} 711$}

A single, narrow $\mathrm{HI}$ absorber is seen at $\Delta v_{\mathrm{abs}}=+129$ $\mathrm{km} \mathrm{s}^{-1}$ with respect to the SDSS group redshift in both Ly $\alpha$ and $\mathrm{Ly} \beta$. A curve-of-growth (CoG) analysis of these lines is poorly constrained, but is consistent with the individual line fits of $\log N_{\mathrm{H} \mathrm{I}}=14.6-15.0$ and $b=20-25 \mathrm{~km} \mathrm{~s}^{-1}$. Despite high$\mathrm{S} / \mathrm{N}$ data, no O VI 1032 absorption is seen corresponding to the $\mathrm{H}$ I lines. A weak Si III line shows that metals are present in this system, but that the ionization state and temperature are likely low. This is consistent with the temperature inferred from the narrow Lyman line profiles $(T \lesssim 80,000 \mathrm{~K})$.

\section{A.2. Group 16803; $\mathrm{z}_{0}=0.14839$, SDSS J1540-0205}

This sight line probes one of the richest groups in our sample $(N=10$ SDSS galaxies; 56 galaxies identified by additional MOS; see Table 8 in Section 4.4) at an impact parameter of $0.5 R_{\text {grp }}$. The background AGN was predicted to have a flux of $\sim 2 \times 10^{-15} \mathrm{erg} \mathrm{s}^{-1} \mathrm{~cm}^{-2} \AA^{-1}$ based on GALEX FUV and NUV fluxes and a detection by Swift/UVOT in the UVW1 channel. However, the observed flux was only about half of this due to a previously unknown partial LLS at the AGN redshift $\left(z_{\mathrm{em}}=0.322\right)$. This means that the continuum level blueward of $\sim 1220 \AA$ is only $\sim 5 \times 10^{-17} \mathrm{erg} \mathrm{s}^{-1} \mathrm{~cm}^{-2} \AA^{-1}$. The data quality around $1400 \AA$ (the region of Ly $\alpha$ at the group redshift) is adequate $(\mathrm{S} / \mathrm{N} \approx 15$ per resel) for the Ly $\alpha$ and $\mathrm{Si}$ III regions. However, $\mathrm{S} / \mathrm{N} \sim 2-3$ for the $\mathrm{O}$ VI and $\mathrm{Ly} \beta$ regions of interest $(\lambda<1200 \AA)$. The only absorption in the redshift range of the group is a single (presumed to be) Ly $\alpha$ line at $1392 \AA$ $\left(\Delta v_{\mathrm{abs}}=-893 \mathrm{~km} \mathrm{~s}^{-1}\right)$. The low data quality at $\lambda<1200 \AA$ precludes stringent limits to $\mathrm{O}$ VI absorption, but no absorption is apparent in either line of the doublet.

\section{A.3. Group 19670; $\mathrm{z}_{0}=0.13443$, SBS $0956+510$}

Strong $\mathrm{H}$ I absorption at $z=0.13388$ is seen in $\operatorname{Ly} \alpha$ and $\operatorname{Ly} \beta$. A CoG solution gives $\log N_{\mathrm{HI}}=15.7$ : and $b=17: \mathrm{km} \mathrm{s}^{-1}$, which fits line profiles reasonably well. A weaker feature at $\lambda=$ $1376.9 \AA$ is identified as a possible Ly $\alpha$ line at $\Delta v_{\mathrm{abs}}=$ $+1069 \mathrm{~km} \mathrm{~s}^{-1}\left(z=0.13267, \log N_{\mathrm{H} \mathrm{I}}=13.1 \pm 0.1, b=29 \pm\right.$ $7 \mathrm{~km} \mathrm{~s}^{-1}$ ), just off the right-hand edge of Figure 1 (online panel 3). No O VI is seen at the redshift of either $\mathrm{HI}$ component. O VI $1032 \AA$ at the SDSS group redshift is blended with $\operatorname{Ly} \beta$ at $z_{\text {abs }}=0.1415$, but no O VI $1038 \AA$ absorption is seen at any redshift.

\section{A.4. Group 25124; $\mathrm{z}_{0}=0.18613$, B $1612+266$}

A strong Ly $\alpha$ absorption line lies very close to the expected group redshift in this sight line $\left(z_{\mathrm{abs}}=0.1853, \Delta v_{\mathrm{abs}}=\right.$ $\left.-212 \mathrm{~km} \mathrm{~s}^{-1}\right)$. Strong, broad O VI $1038 \AA$ absorption is detected $\left(\log N_{\mathrm{O} \text { VI }}=14.49 \pm 0.05, b=54 \pm 6 \mathrm{~km} \mathrm{~s}^{-1}\right)$ at a slightly higher redshift (see Section 3.2), but the stronger O VI $1032 \AA$ line is blended with the H I Ly10 line of a partial LLS at $z_{\mathrm{abs}}=0.3306$.

We analyze the blended line in two ways: first, we use the unblended HI lines of the partial LLS to model the column density and $b$-value of the system; Ly 6-9 and 11-15 are clearly present in the data and show no obvious blending with other systems. A CoG analysis gives a solution of $\log N_{\mathrm{HI}}=16.60 \pm 0.02, b=27 \pm 2 \mathrm{~km} \mathrm{~s}^{-1}$.

With this information, we model the partial LLS absorption from the data and recover the O VI $1032 \AA$ line, which appears consistent with the weaker $1038 \AA$ line. We check this for consistency with a second method that uses the observed O VI $1038 \AA$ line as a model and subtracts it from the data near the O VI+Ly10 blend at $1223 \AA$ A, yielding a Ly10 profile consistent with the CoG solution.

In addition to $\mathrm{H} \mathrm{I} \mathrm{Ly} \alpha$ and $\mathrm{O}$ VI, corresponding absorption is also seen in Si III 1206.5 and Si II 989, 1190, 1193 at $z_{\mathrm{abs}}=0.1853$. Corresponding $\operatorname{Ly} \beta$ absorption is obscured in the Galactic Ly $\alpha$ profile and is unrecoverable. This system is discussed in detail in Section 3.2. 
Table 10

Absorption Lines Measured within 2.5 $\sigma_{0}$ of SDSS Group Velocities

\begin{tabular}{|c|c|c|c|c|c|c|}
\hline Species & $z_{\mathrm{abs}}$ & $\underset{\left(\mathrm{km} \mathrm{s}^{-1}\right)}{\Delta v_{\mathrm{abs}}}$ & $\begin{array}{l}\text { S.L. } \\
(\sigma)\end{array}$ & $\begin{array}{l}\mathrm{EW}_{r} \\
(\mathrm{~mA})\end{array}$ & $\begin{array}{c}b \\
\left(\mathrm{~km} \mathrm{~s}^{-1}\right)\end{array}$ & $\begin{array}{c}\log N \\
\left(N \text { in } \mathrm{cm}^{-2}\right)\end{array}$ \\
\hline \multicolumn{7}{|c|}{ RBS711, Group \#12833; $z_{0}=0.14693$} \\
\hline Ly $\alpha 1215$ & 0.14743 & 129 & 42.8 & $333 \pm 33$ & $23 \pm 2$ & $14.65 \pm 0.19$ \\
\hline Ly $\beta 1025$ & 0.14743 & 131 & 31.7 & $241 \pm 14$ & $24 \pm 1$ & $15.03 \pm 0.07$ \\
\hline Si III 1206 & 0.14742 & 127 & 6.9 & $42 \pm 10$ & $15 \pm 4$ & $12.37 \pm 0.09$ \\
\hline \multicolumn{7}{|c|}{ SDSS J15403-0205, Group \#16803; $z_{0}=0.14839^{\mathrm{a}}$} \\
\hline Ly $\alpha 1215$ & 0.14497 & -893 & 7.1 & $110 \pm 17$ & $27 \pm 5$ & $13.41 \pm 0.07$ \\
\hline \multicolumn{7}{|c|}{ SBS0956+509, Group $\# 19670 ; z_{0}=0.13443$} \\
\hline Ly $\alpha 1215$ & 0.13388 & -146 & 30.7 & $335 \pm 26$ & $25 \pm 2$ & $14.49 \pm 0.12$ \\
\hline Ly $\alpha 1215$ & 0.13848 & 1069 & 5.3 & $51 \pm 9$ & $38 \pm 8$ & $13.01 \pm 0.07$ \\
\hline Ly $\beta 1025$ & 0.13387 & -146 & 20.8 & $233 \pm 15$ & $26 \pm 2$ & $14.90 \pm 0.05$ \\
\hline \multicolumn{7}{|c|}{ QSOB1612 +266 , Group $\# 25124 ; z_{0}=0.18613$} \\
\hline Ly $\alpha 1215$ & 0.18529 & -212 & 49.7 & $705 \pm 77$ & $37 \pm 3$ & $15.81 \pm 0.33$ \\
\hline Ly $\alpha 1215$ & 0.18569 & -110 & 9.0 & $120 \pm 10$ & $32 \pm 4$ & $13.44 \pm 0.05$ \\
\hline O VI 1032 & 0.18540 & -184 & 13.0 & $248 \pm 90$ & $53 \pm 4$ & $14.49 \pm 0.05$ \\
\hline O VI 1038 & 0.18540 & -184 & 13.0 & $160 \pm 21$ & $53 \pm 4$ & $14.49 \pm 0.05$ \\
\hline Si III 1206 & 0.18517 & -242 & 9.7 & $150 \pm 25$ & $59 \pm 10$ & $12.92 \pm 0.08$ \\
\hline Si III 1206 & 0.18531 & -208 & 32.7 & $364 \pm 22$ & $30 \pm 2$ & $13.80 \pm 0.07$ \\
\hline C III 977 & 0.18520 & -234 & 9.5 & $289 \pm 129$ & $38 \pm 11$ & $13.98 \pm 0.45$ \\
\hline C III 977 & 0.18537 & -191 & 13.7 & $323 \pm 83$ & $21 \pm 4$ & $15.32 \pm 0.72$ \\
\hline N III 989 & 0.18543 & -176 & 14.8 & $215 \pm 21$ & $34 \pm 3$ & $14.54 \pm 0.05$ \\
\hline Si II 1190 & 0.18529 & -212 & 13.9 & $166 \pm 15$ & $33 \pm 2$ & $13.79 \pm 0.04$ \\
\hline Si II 1193 & 0.18531 & -207 & 16.5 & $199 \pm 103$ & $28 \pm 1$ & $13.65 \pm 0.02$ \\
\hline \multicolumn{7}{|c|}{ SDSSJ133300.83+451809.0, Group \#32123; $z_{0}=0.15971$} \\
\hline Ly $\alpha 1215$ & 0.15874 & -251 & 37.1 & $643 \pm 67$ & $41 \pm 4$ & $15.10 \pm 0.22$ \\
\hline Ly $\alpha 1215$ & 0.16081 & 283 & 15.7 & $234 \pm 20$ & $22 \pm 2$ & $14.02 \pm 0.07$ \\
\hline Ly $\alpha 1215$ & 0.16142 & 442 & 4.5 & $95 \pm 30$ & $49 \pm 13$ & $13.29 \pm 0.11$ \\
\hline Ly $\beta 1025$ & 0.15860 & -285 & 10.1 & $136 \pm 32$ & $15 \pm 4$ & $14.67 \pm 0.15$ \\
\hline $\operatorname{Ly} \beta 1025$ & 0.15887 & -218 & 16.2 & $295 \pm 33$ & $32 \pm 4$ & $15.03 \pm 0.07$ \\
\hline $\operatorname{Ly} \beta 1025$ & 0.16090 & 306 & 4.8 & $46 \pm 15$ & $11 \pm 6$ & $13.94 \pm 0.13$ \\
\hline O VI 1032 & 0.16088 & 302 & 2.3 & $36 \pm 15$ & $29 \pm 13$ & $13.55 \pm 0.25$ \\
\hline O VI 1038 & 0.16082 & 288 & 3.0 & $44 \pm 35$ & $33 \pm 16$ & $13.89 \pm 0.28$ \\
\hline Si III 1206 & 0.15883 & -227 & 6.0 & $77 \pm 16$ & $15 \pm 4$ & $12.69 \pm 0.09$ \\
\hline Si III 1206 & 0.16079 & 278 & 2.9 & $24 \pm 11$ & $5 \pm 0$ & $12.19 \pm 0.21$ \\
\hline \multicolumn{7}{|c|}{ SDSSJ10282+2119, Group \#36001; $z_{0}=0.18788$} \\
\hline Ly $\alpha 1215$ & 0.18429 & -906 & 35.3 & $507 \pm 32$ & $38 \pm 2$ & $14.68 \pm 0.09$ \\
\hline $\operatorname{Ly} \alpha 1215$ & 0.18494 & -743 & 5.5 & $46 \pm 25$ & $17 \pm 11$ & $13.00 \pm 0.22$ \\
\hline Ly $\alpha 1215$ & 0.18527 & -659 & 44.8 & $523 \pm 106$ & $28 \pm 5$ & $15.56 \pm 0.62$ \\
\hline $\operatorname{Ly} \alpha 1215$ & 0.18585 & -513 & 27.9 & $367 \pm 43$ & $26 \pm 2$ & $14.63 \pm 0.18$ \\
\hline $\mathrm{Ly} \gamma 972$ & 0.18526 & -660 & 18.7 & $344 \pm 12$ & $18 \pm 0$ & $18.00 \pm 0.00$ \\
\hline $\mathrm{Ly} \gamma 972$ & 0.18592 & -494 & 8.5 & $172 \pm 28$ & $23 \pm 5$ & $15.16 \pm 0.09$ \\
\hline O VI 1032 & 0.18436 & -887 & 4.1 & $42 \pm 36$ & $32 \pm 10$ & $13.56 \pm 0.43$ \\
\hline O VI 1032 & 0.18530 & -651 & 3.6 & $30 \pm 1$ & $23 \pm 9$ & $13.43 \pm 0.13$ \\
\hline O VI 1038 & 0.18432 & -898 & 3.6 & $24 \pm 2$ & $18 \pm 8$ & $13.63 \pm 0.17$ \\
\hline Si III 1206 & 0.18527 & -658 & 8.1 & $90 \pm 21$ & $29 \pm 4$ & $12.71 \pm 0.09$ \\
\hline C III 977 & 0.18532 & -646 & 3.8 & $65 \pm 24$ & $22 \pm 7$ & $13.10 \pm 0.14$ \\
\hline C III 977 & 0.18604 & -464 & 4.4 & $111 \pm 38$ & $66 \pm 14$ & $13.29 \pm 0.07$ \\
\hline \multicolumn{7}{|c|}{ CSO1022, Groups \#44564, 44565; $z_{0}=0.14506,0.14925$ [no absorbers] } \\
\hline \multicolumn{7}{|c|}{ CSO1080, Group \#44726; $z_{0}=0.15208$ [no absorbers] } \\
\hline \multicolumn{7}{|c|}{ FBQSJ1519+2838, Group \#44739; $z_{0}=0.11839$} \\
\hline Ly $\alpha 1215$ & 0.11537 & -808 & 46.6 & $497 \pm 40$ & $32 \pm 2$ & $14.98 \pm 0.21$ \\
\hline $\operatorname{Ly} \alpha 1215$ & 0.11706 & -357 & 36.7 & $436 \pm 39$ & $30 \pm 2$ & $14.78 \pm 0.17$ \\
\hline Ly $\alpha 1215$ & 0.11766 & -195 & 35.6 & $509 \pm 48$ & $37 \pm 3$ & $14.72 \pm 0.11$ \\
\hline Ly $\alpha 1215$ & 0.11795 & -118 & 17.2 & $191 \pm 26$ & $21 \pm 3$ & $13.84 \pm 0.09$ \\
\hline $\mathrm{Ly} \beta 1025$ & 0.11535 & -814 & 19.9 & $325 \pm 30$ & $32 \pm 3$ & $15.20 \pm 0.12$ \\
\hline $\mathrm{Ly} \beta 1025$ & 0.11704 & -362 & 26.2 & $290 \pm 54$ & $25 \pm 4$ & $15.34 \pm 0.28$ \\
\hline Ly $\beta 1025$ & 0.11762 & -205 & 20.8 & $304 \pm 30$ & $31 \pm 3$ & $15.11 \pm 0.09$ \\
\hline $\mathrm{Ly} \beta 1025$ & 0.11793 & -123 & 6.1 & $77 \pm 21$ & $22 \pm 7$ & $14.13 \pm 0.11$ \\
\hline O VI 1032 & 0.11551 & -773 & 3.2 & $26 \pm 19$ & $10 \pm 10$ & $13.39 \pm 0.33$ \\
\hline O VI 1032 & 0.11766 & -197 & 3.6 & $49 \pm 27$ & $40 \pm 15$ & $13.63 \pm 0.18$ \\
\hline Si III 1206 & 0.11526 & -839 & 4.9 & $85 \pm 31$ & $64 \pm 15$ & $12.64 \pm 0.12$ \\
\hline Si III 1206 & 0.11709 & -348 & 4.6 & $30 \pm 29$ & $5 \pm 0$ & $12.32 \pm 0.12$ \\
\hline Si III 1206 & 0.11765 & -197 & 5.2 & $53 \pm 12$ & $17 \pm 4$ & $12.48 \pm 0.09$ \\
\hline
\end{tabular}


Table 10

(Continued)

\begin{tabular}{|c|c|c|c|c|c|c|}
\hline Species & $z_{\mathrm{abs}}$ & $\underset{\left(\mathrm{km} \mathrm{s}^{-1}\right)}{\Delta v_{\mathrm{abs}}}$ & $\begin{array}{l}\text { S.L. } \\
(\sigma)\end{array}$ & $\begin{array}{l}\mathrm{EW}_{r} \\
(\mathrm{~mA})\end{array}$ & $\begin{array}{c}b \\
\left(\mathrm{~km} \mathrm{~s}^{-1}\right)\end{array}$ & $\begin{array}{c}\log N \\
\left(N \text { in } \mathrm{cm}^{-2}\right)\end{array}$ \\
\hline Ly $\alpha 1215$ & 0.12551 & -501 & 61.2 & $581 \pm 166$ & $29 \pm 6$ & $15.94 \pm 0.96$ \\
\hline Ly $\alpha 1215$ & 0.12567 & -460 & 32.4 & $372 \pm 189$ & $43 \pm 13$ & $14.12 \pm 0.49$ \\
\hline Ly $\beta 1025$ & 0.12550 & -504 & 30.8 & $365 \pm 75$ & $30 \pm 5$ & $15.57 \pm 0.37$ \\
\hline Ly $\beta 1025$ & 0.12568 & -457 & 20.6 & $211 \pm 147$ & $21 \pm 28$ & $15.00 \pm 2.36$ \\
\hline O VI 1032 & 0.12571 & -449 & 9.8 & $93 \pm 12$ & $21 \pm 3$ & $14.01 \pm 0.06$ \\
\hline O VI 1038 & 0.12571 & -450 & 5.1 & $52 \pm 15$ & $20 \pm 6$ & $14.00 \pm 0.10$ \\
\hline \multicolumn{7}{|c|}{ FBQSJ103059.1+310255, Group \#50433; $z_{0}=0.13599$ [no absorbers] } \\
\hline
\end{tabular}

Note. Column 3 lists the velocity offset between the absorber and the SDSS group redshift in Table 2, and Column 4 lists the significance level of the detected absorption.

${ }^{\mathrm{a}} \mathrm{O}$ VI in this sight line is obscured by a partial LLS at $z=0.322$.

\section{A.5. Group 32123; $\mathrm{z}_{0}=0.15971$, SDSS J1333+4518}

Several strong absorbers are present in this sight line at $v_{\text {grp }} \pm 500 \mathrm{~km} \mathrm{~s}^{-1}$, though none at $\left|\Delta v_{\mathrm{abs}}\right|<200 \mathrm{~km} \mathrm{~s}^{-1}$. Strong $\operatorname{Ly} \alpha$ systems are seen at $z=0.15874$ and $z=$ 0.16081 , bracketing the SDSS group redshift. The blue system shows two components in $\mathrm{Ly} \beta$, while the red system shows a single $\mathrm{Ly} \beta$ component along with weak O VI absorption in both lines of the doublet. Both red and blue absorbers show weak $\mathrm{Si}$ III absorption, though the red $\mathrm{Si}$ III detection is marginal $(\sim 2.8 \sigma)$. A possible metal-free BLA is in this group at $z=0.16142\left(\Delta v_{\mathrm{abs}}=+442 \mathrm{~km} \mathrm{~s}^{-1}\right)$.

\section{A.6. Group $36001 ; \mathrm{z}_{0}=0.18788$, SDSS J1028+2119}

While there is no absorption at the SDSS group redshift, three strong $\operatorname{Ly} \alpha$ lines are found at $\Delta v_{\mathrm{abs}}=-1100$ to $-500 \mathrm{~km} \mathrm{~s}^{-1}$. The first absorber at $z=0.18429\left(\Delta v_{\mathrm{abs}}=\right.$ $-906 \mathrm{~km} \mathrm{~s}^{-1}$ ) is outside the relative velocity bounds established for association with this group. There is also weak O VI 1031, $1038 \AA$ absorption associated with the $z=0.18527$ Ly $\alpha$ absorber. Relative to the SDSS group centroid and virial radius, this sight line has the smallest impact parameter $\left(\rho_{*}=\right.$ $295 \mathrm{kpc}$ ) in our sample.

\section{A.7. Groups 44565, 44564; $\mathrm{z}_{0}=0.14925,0.14506$, CSO 1022}

This archival data set probes two SDSS groups $\sim 1200 \mathrm{~km} \mathrm{~s}^{-1}$ apart, which were shown to be parts of a single, much more massive group (see Appendix B). While a low-significance feature $(2.8 \sigma)$ is consistent with O VI $1032 \AA$ absorption at the redshift of group 44564, it is identified as O VI $1038 \AA$ absorption from the unrelated, strong $z=0.1387$ system (confirmed via $\mathrm{H}$ I and other metal lines at $z=0.1387$ ). No absorption is seen in $\mathrm{H}$ I through either Ly $\alpha$ or $\operatorname{Ly} \beta$ near the redshift of either group. The group characterization analysis discussed in Section 4.4 merges these two groups into one, with velocity centroid between the values provided and sky position very close to the centroids of the two SDSS groups.

\section{A.8. Group 44726; $\mathrm{z}_{0}=0.15208$, CSO 1080}

The long IGM sight line $\left(z_{\mathrm{em}}=0.526\right)$ toward this background AGN provides a complicated absorption-line population against which we must measure absorption from the group. Nevertheless, there is no H I absorption within
$\left|\Delta v_{\text {abs }}\right|<1000 \mathrm{~km} \mathrm{~s}^{-1}$ of the SDSS group redshift, though a strong $\mathrm{H}$ I system is found at $\Delta v_{\mathrm{abs}}=+1300 \mathrm{~km} \mathrm{~s}^{-1}$.

\section{A.9. Groups 44739, 44858; $\mathrm{z}_{0}=0.11839,0.12740$, FBQS J1519+2838}

At the redshift of group $44739\left(z_{0}=0.1184\right)$, four strong H I lines are seen in both $\operatorname{Ly} \alpha$ and $\operatorname{Ly} \beta$ between -800 and $-100 \mathrm{~km} \mathrm{~s}^{-1}$ from the SDSS group redshift. Two marginal O VI 1032 lines are seen which appear to correspond with the -808 and $-195 \mathrm{~km} \mathrm{~s}^{-1}$ components. The potentially broad O VI $1032 \AA$ detection is weak enough that it is consistent with a non-detection in the $1038 \AA$ line. There is Si III absorption seen corresponding to three of the H I components, as well.

For group $44858\left(z_{0}=0.1274\right)$, a single, strong $\mathrm{H}$ I absorber is best-fit as a pair of Ly $\alpha$ lines at $\Delta v_{\text {abs }}=-501$ and $-460 \mathrm{~km} \mathrm{~s}^{-1}$ with respect to the SDSS group redshift. Moderate, narrow O VI absorption is seen in both lines of the doublet at very nearly the $\Delta v_{\text {abs }}=-460 \mathrm{~km} \mathrm{~s}^{-1}$ velocity. Si III at this redshift is blended with another intervening system, and no other commonly seen low-ionization species are present in these data.

\section{A.10. Group 50433; $\mathrm{z}_{0}=0.13599 ; \mathrm{FBQS} \mathrm{J1030+3102}$}

High-S/N data at the expected $\mathrm{O}$ VI, $\operatorname{Ly} \alpha$, and $\operatorname{Ly} \beta$ line locations show no absorption features in $\mathrm{HI}$ or $\mathrm{O}$ VI within $\pm 1500 \mathrm{~km} \mathrm{~s}^{-1}$ of the group velocity.

\section{Appendix B \\ Notes on Properties of Individual Groups}

Figure 15 examines how the derived properties of the galaxy groups are related. These properties show a high level of discretization for the sparsest groups $\left(N_{\text {grp }} \lesssim 10\right)$, as well as strong correlations with $M_{\mathrm{grp}}$ through Equations (5)-(6). Furthermore, there are often peaks for poor, low-mass and rich, high-mass configurations for a given group. Nevertheless, the group properties in Table 8 are almost always within the $1 \sigma$ range of values derived from the individual Monte Carlo realizations (Table 6), indicating that the adopted group configurations are representative of the ensemble as a whole. This is reassuring but not surprising, as we adopt the configuration that most closely matches the marginal distributions of these parameters (Section 4.4). In the following discussion, we examine the properties of individual groups. 


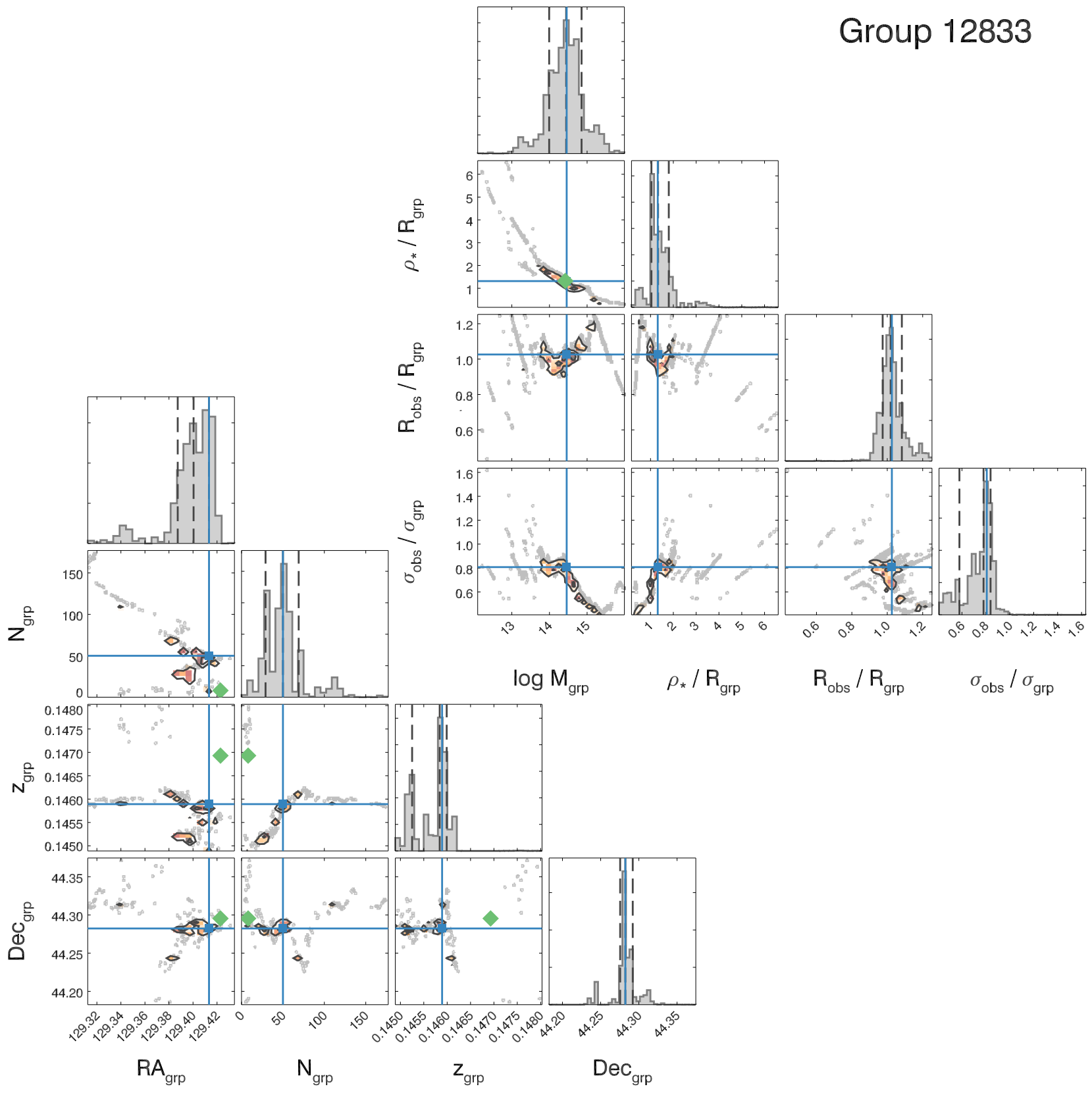

Figure 15. Exploration of parameter covariance for group 12833. Contours are drawn at $1 \sigma$ and $2 \sigma$ significance. Inside the $2 \sigma$ contour, data are color-coded according to density, with lighter colors indicating lower density. Outside the $2 \sigma$ contour, data from individual Monte Carlo realizations are shown as gray circles. Dashed lines in the histograms show the 16th, 50th, and 84th percentile values (i.e., the ranges listed in Table 6). Solid blue lines show the adopted values from Table 8 (see Section 4.4). Green diamonds show the values from the SDSS analysis in Table 2, where applicable.

(The complete figure set (12 images) is available.)

\section{B.1. Group 12833}

The vast majority of the Monte Carlo realizations for this group have $<80$ members (Table 6 ), but there is a small tail up to $\sim 150$ members (Figure 15). The group position is well constrained in decl., but has two peaks in R.A.; the group redshift also shows two peaks separated by $\sim 250 \mathrm{~km} \mathrm{~s}^{-1}$. The group mass shows a strong peak at $\sim 10^{14.5} M_{\odot}$ and a weaker one at $\sim 10^{13} M_{\odot}$, and the impact parameter of the QSO is well constrained at $\rho_{\star} \approx 1.3 R_{\mathrm{grp}}$. The distributions of $R_{\mathrm{obs}} / R_{\mathrm{grp}}$ and $\sigma_{\text {obs }} / \sigma_{\text {grp }}$ are fairly broad, which is a general characteristic for all groups, owing to the noisy nature of $R_{\mathrm{obs}}$ and $\sigma_{\mathrm{obs}}$. The adopted group properties (blue lines) all fall within the $1 \sigma$ uncertainty.

\section{B.2. Group 16803}

The properties of this group are all reasonably well constrained (Figure 15, online panel 2), except for the $R_{\mathrm{obs}} / R_{\mathrm{grp}}$ and $\sigma_{\mathrm{obs}} / \sigma_{\mathrm{grp}}$ values, whose marginal likelihoods have multiple peaks. The adopted group properties are all near the peaks of the marginal distributions. 


\section{B.3. Group 19670}

Most of the derived properties of this group show evidence for multiple peaks (Figure 15, online panel 3), suggesting that a relatively low-mass, $\sim 50$-member group and a higher-mass, $\sim 90$-member group are both common outcomes. The adopted group properties favor the lower-mass configuration, and all fall within the $1 \sigma$ uncertainty.

\section{B.4. Group 25124}

This is perhaps the hardest group in our sample to characterize. The most common configurations (Figure 15, online panel 4) are a $\sim 30$-member, low-mass $\left(\sim 10^{14.4} M_{\odot}\right)$ group, and a $\sim 40$-member, high-mass $\left(\sim 10^{14.8} M_{\odot}\right)$ group; however, the richness distribution for the high-mass configuration is broad and extends up to $\sim 50$ members. The group redshift distribution shows two peaks separated by $\sim 300 \mathrm{~km} \mathrm{~s}^{-1}$, and the $R_{\mathrm{obs}} / R_{\mathrm{grp}}$ and $\sigma_{\mathrm{obs}} / \sigma_{\mathrm{grp}}$ distributions are also multi-modal. Confoundingly, the two group configurations evident in the richness and mass distributions do not correspond to two clear positions on the sky; instead there are 5 fairly common group positions in the Monte Carlo realizations. Our adopted group membership favors the most common of these positions, which corresponds to one of the lowmass group configurations.

\section{B.5. Group 32123}

The Monte Carlo realizations for this group tend to find $\lesssim 20$ members (Figure 15, online panel 5), but there is a tail that extends up to $\sim 50$ members. The group position on the sky shows a clearly favored position, but the group redshift is more widely distributed; the adopted group configuration matches the richness and favored position well. The mass and other derived properties also have multiple peaks in their distributions; the adopted group values are all in the high-density regions for these properties.

\section{B.6. Group 36001}

The Monte Carlo realizations all find a modest $(<15$ member) group, with a six-member configuration being the most common (Figure 15, online panel 6). Due to the small number of galaxies involved, the group position and redshift distributions tend to settle on a handful of discrete values. The mass and other derived properties show smoother distributions, due to the uncertainty in $\Upsilon_{\text {grp }}$ that is folded into the Monte Carlo process (see Section 4.3). The adopted group properties match the peaks in all of these quantities.

\section{B.7. Groups 44564 and 44565}

These two groups are both probed by the same QSO sight line (Table 1), and the initial SDSS analysis found them to be separated by 1.8 on the sky and $1100 \mathrm{~km} \mathrm{~s}^{-1}$ in redshift (Table 2). We find many galaxies at intermediate redshifts in this region of the sky, so the two SDSS groups merge into a single, $\sim 115$-member group by our analysis. The Monte Carlo realizations find positions and redshifts for the groups separated by $\sim 10^{\prime \prime}$ on the sky and $\sim 5 \mathrm{~km} \mathrm{~s}^{-1}$ in redshift (Table 6), and all other derived (and adopted) group quantities are also nearly identical. The adopted position of the merged group is closer to the SDSS position of group 44565, but the adopted redshift is closer to the SDSS redshift of group 44564 (Table 8).

\section{B.8. Group 44726}

The vast majority of the Monte Carlo realizations find a modest group with $<30$ members, but there is a tail that extends all the way up to $>150$ members (Figure 15, online panel 9). This leads to a bimodality in the inferred mass and other derived quantities. Our adopted group properties lie near the peaks of all of the parameter distributions and prefer a lowmass $\left(\sim 10^{13.4} M_{\odot}\right)$ group. The adopted seven-member group is also remarkably compact, with $R_{\text {obs }}<200 \mathrm{kpc}$ (Table 8).

\section{B.9. Group 44739}

Groups 44739 and 44858 are probed by the same QSO sight line (Table 1). However, the initial SDSS analysis found them to be much more separated $\left(30^{\prime}\right.$ on the sky and $2400 \mathrm{~km} \mathrm{~s}^{-1}$ in redshift; Table 2) than Groups 44564 and 44565, so they do not merge into a single, larger group by our analysis. Thus, we continue to discuss their properties separately.

Most of the Monte Carlo realizations find groups with $\lesssim 100$ members, but occasionally they will merge this group with Group 44858, which allows the group to grow to several hundred members (Figure 15, online panel 10). Ignoring this low-frequency tail, the group position, richness, and redshift are well constrained. The inferred mass and other derived group properties are also well-behaved. The adopted group properties are all near the peaks of the marginal distributions.

\section{B.10. Group 44858}

As with Group 44739, most of the Monte Carlo realizations have $<100$ members, but there is a low-frequency tail that extends up to $\sim 600$ members when the two groups occasionally merge (Figure 15, online panel 11). Outside of these rare cases, the group properties are well constrained. The adopted group properties match the peaks of the marginal distributions, except for $R_{\mathrm{obs}} / R_{\mathrm{grp}}$ and $\sigma_{\mathrm{obs}} / \sigma_{\mathrm{grp}}$, which lie outside the $1 \sigma$ uncertainty.

\section{B.11. Group 50433}

The Monte Carlo realizations almost always find $<40$ members in this group (Figure 15, online panel 12), with several discrete positions, richnesses, and redshifts. The distributions of inferred mass and other derived quantities are smooth and well-behaved, and the adopted group properties are near the peaks of all of these quantities.

\section{ORCID iDs}

Brian A. Keeney (1) https://orcid.org/0000-0003-0797-5313 Charles W. Danforth (1D https://orcid.org/0000-00034738-6601

Benjamin D. Oppenheimer (1) https://orcid.org/0000-00023391-2116

Andreas A. Berlind (iD https://orcid.org/0000-0002-1814-2002

\section{References}

Adelberger, K. L., Shapley, A. E., Steidel, C. C., et al. 2005, ApJ, 629, 636 Adhikari, S., Dalal, N., \& Chamberlain, R. T. 2014, JCAP, 11, 19 Beers, T. C., Flynn, K., \& Gebhardt, K. 1990, AJ, 100, 32

Berlind, A., Frieman, J., Weinberg, D. H., et al. 2006, ApJS, 167, 1

Binney, J., \& Tremaine, S. 1987, Galactic Dynamics (Princeton, NJ: Princeton Univ. Press)

Bordoloi, R., Tumlinson, J., Werk, J. K., et al. 2014, ApJ, 796, 136 
Borthakur, S., Heckman, T., Strickland, D., Wild, V., \& Schiminovich, D. 2013, ApJ, 768, 18

Burchett, J. N., Tripp, T. M., Bordoloi, R., et al. 2016, ApJ, 832, 124

Cen, R., \& Ostriker, J. P. 1999, ApJ, 514, 1

Chen, H.-W., Lanzetta, K. M., Webb, J. K., \& Barcons, X. 2001, ApJ, 559, 654 Chilingarian, I. V., Melchior, A.-L., \& Zolotukhin, I. Y. 2010, MNRAS, 405, 1409

Chilingarian, I. V., \& Zolotukhin, I. Y. 2012, MNRAS, 419, 1727

Danforth, C. W., \& Shull, J. M. 2008, ApJ, 679, 194

Danforth, C. W., Keeney, B. A., Tilton, E. M., et al. 2016, ApJ, 817, 111

Davé, R., Hernquist, L., Katz, N., \& Weinberg, D. H. 1999, ApJ, 511, 521

Diemer, B. 2017, ApJS, 231, 5

Diemer, B., \& Kravtsov, A. V. 2014, ApJ, 789, 1

Fabricant, D., Fata, R., Roll, J., et al. 2005, PASP, 117, 1411

Hinshaw, G., Larson, D., Komatsu, E., et al. 2013, ApJS, 208, 19

Johnson, S. D., Chen, H.-W., \& Mulchaey, J. S. 2015, MNRAS, 449, 3263

Keeney, B. A., Danforth, C. W., Stocke, J. T., France, K., \& Green, J. C. 2012, PASP, 124, 830

Keeney, B. A., Momjian, E., Stocke, J. T., Carilli, C. L., \& Tumlinson, J. 2005, ApJ, 622, 267

Keeney, B. A., Pratt, C. T., Stocke, J. T., et al. 2018, ApJS, 237, 11

Keeney, B. A., Stocke, J. T., Danforth, C. W., et al. 2017, ApJS, 230, 6

Klypin, A., Kravtsov, A. V., Bullock, J. S., \& Primack, J. R. 2001, ApJ, 554, 903

Klypin, A., Kravtsov, A. V., Valenzuela, O., \& Prada, F. 1999, ApJ, 522, 82

Le Brun, A. M. C., McCarthy, I. G., Schaye, J., \& Ponman, T. J. 2014, MNRAS, 441, 1270

Lehner, N., Howk, J. C., \& Wakker, B. P. 2015, ApJ, 804, 79

Lehner, N., O’Meara, J. M., Fox, A. J., et al. 2014, ApJ, 788, 119

McCarthy, I. G., Schaye, J., Bird, S., \& Le Brun, A. M. C. 2017, MNRAS, 465, 2936

McGaugh, S. S., Schombert, J. M., Bothun, G. D., \& de Blok, W. J. G. 2000, ApJL, 533, L99

Montero-Dorta, A. D., \& Prada, F. 2009, MNRAS, 399, 1106

More, S., Diemer, B., \& Kravtsov, A. V. 2015, ApJ, 810, 36

Mulchaey, J. S. 2000, ARA\&A, 38, 289

Mulchaey, J. S., Davis, D. S., Mushotzky, R. F., \& Burstein, D. 1996, ApJ, 456, 80

Murray, S., Power, C., \& Robotham, A. 2013, A\&C, 3, 23

Nelson, D., Kauffmann, G., Pillepich, A., et al. 2018, MNRAS, 447, 450

Nicastro, F., Kaastra, J., Krongold, Y., et al. 2018, Natur, 558, 406

Oppenheimer, B. D., Davé, R., Katz, N., Kollmeier, J. A., \& Weinberg, D. H. 2012, MNRAS, 420, 829
Osmond, J. P. F., \& Ponman, T. J. 2004, MNRAS, 350, 1511

Pagel, B. E. J. 2009, Nucleosynthesis and Chemical Evolution of Galaxies (Cambridge: Cambridge Univ. Press)

Pisani, A., Ramella, M., \& Geller, M. J. 2003, AJ, 126, 1677

Planck Collaboration, Ade, P. A. R., Aghanim, N., et al. 2014, A\&A, 571, A16

Pratt, C. T., Stocke, J. T., Keeney, B. A., \& Danforth, C. W. 2018, ApJ, 855,18

Prochaska, J. X., Weiner, B., Chen, H.-W., Mulchaey, J., \& Cooksey, K. 2011, ApJ, 740, 91

Prochaska, J. X., Werk, J. K., Worseck, G., et al. 2017, ApJ, 837, 169

Proctor, R. N., Mendes de Oliveira, C., Azanha, L., Dupke, R., \& Overzier, R. 2015, MNRAS, 449, 2345

Rauch, M. 1998, ARA\&A, 36, 267

Savage, B. D., Kim, T.-S., Keeney, B., et al. 2012, ApJ, 753, 80

Savage, B. D., Kim, T.-S., Wakker, B. P., et al. 2014, ApJS, 212, 8

Savage, B. D., Narayanan, A., Wakker, B. P., et al. 2010, ApJ, 719, 1526

Schaye, J., Crain, R. A., Bower, R. G., et al. 2015, MNRAS, 446, 521

Schneider, D. P., Hartig, G. F., Jannuzi, B. T., et al. 1993, ApJS, 87, 45

Shull, J. M., Smith, B. D., \& Danforth, C. W. 2012, ApJ, 759, 23

Steidel, C. C., Erb, D. K., Shapley, A. E., et al. 2010, ApJ, 717, 289

Stocke, J. T., Keeney, B. A., \& Danforth, C. W. 2010, PASA, 27, 256

Stocke, J. T., Keeney, B. A., Danforth, C. W., et al. 2013, ApJ, 763, 148

Stocke, J. T., Keeney, B. A., Danforth, C. W., et al. 2014, ApJ, 791, 128

Stocke, J. T., Keeney, B. A., Danforth, C. W., et al. 2017, ApJ, 838, 37

Stocke, J. T., Penton, S. V., Danforth, C. W., et al. 2006, ApJ, 641, 217

Tejos, N., Rodróuez-Puebla, A., \& Primack, J. R. 2018, MNRAS, 473, 366

Terrell, D. G., \& Scott, D. W. 1992, AnSta, 20, 1236

Thom, C., Tumlinson, J., Werk, J. K., et al. 2012, ApJL, 758, L41

Tinker, J., Kravtsov, A. V., Klypin, A., et al. 2008, ApJ, 688, 709

Tremonti, C. A., Heckman, T. M., Kauffmann, G., et al. 2004, ApJ, 613, 898

Tumlinson, J., Giroux, M. L., Shull, J. M., \& Stocke, J. T. 1999, AJ, 118, 2148

Tumlinson, J., Thom, C., Werk, J. K., et al. 2011, Sci, 334, 948

Tumlinson, J., Thom, C., Werk, J. K., et al. 2013, ApJ, 777, 59

Wakker, B. P., Hernandez, A. K., French, D. M., et al. 2015, ApJ, 814, 40

Werk, J. K., Prochaska, J. X., Thom, C., et al. 2013, ApJS, 204, 17

Werk, J. K., Prochaska, J. X., Tumlinson, J., et al. 2014, ApJ, 792, 8

Wu, X-P., Xue, Y-J., \& Fang, L-Z. 1999, ApJ, 524, 22

Yoon, J. H., \& Putman, M. E. 2013, ApJL, 772, L29

Yoon, J. H., \& Putman, M. E. 2017, ApJ, 839, 117

Yoon, J. H., Putman, M. E., Thom, C., Chen, H.-W., \& Bryan, G. L. 2012 ApJ, 754, 84 